EUROPEAN ORGANISATION FOR PARTICLE PHYSICS

CERN-PPE/96-074

5 June, 1996

\title{
A Measurement of the $B_{d}^{0}$ Oscillation Frequency Using Leptons and $\mathrm{D}^{* \pm}$ Mesons
}

\author{
The OPAL Collaboration
}

\begin{abstract}
Data collected with the OPAL detector during 1990-1994 are used to measure the time dependence of $\mathrm{B}_{\mathrm{d}}^{0} \leftrightarrow \overline{\mathrm{B}}_{\mathrm{d}}^{0}$ mixing. A sample of $348 \mathrm{D}^{* \pm}$ candidates with a lepton in the opposite hemisphere are reconstructed, of which $167 \pm 25$ are expected to be from $\mathrm{B}_{\mathrm{d}}^{0}$ decays. The $\mathrm{B}_{\mathrm{d}}^{0}$ oscillation frequency is measured to be

$$
\Delta m_{\mathrm{d}}=0.567 \pm 0.089(\mathrm{stat})_{-0.023}^{+0.029}(\mathrm{syst}) \mathrm{ps}^{-1} \text {. }
$$

A previously published analysis of $\Delta m_{\mathrm{d}}$ using $\mathrm{D}^{* \pm}$ and lepton candidates in the same hemisphere and jet charge is also updated with a larger data sample. From $1200 \mathrm{D}^{* \pm} \ell^{\mp}$ candidates, of which $778 \pm 84$ are expected to be from $\mathrm{B}_{\mathrm{d}}^{0}$ decays, we find a value of:

$$
\Delta m_{\mathrm{d}}=0.539 \pm 0.060 \text { (stat) } \pm 0.024 \text { (syst) } \mathrm{ps}^{-1} \text {. }
$$
\end{abstract}

The combined result of these two analyses is

$$
\Delta m_{\mathrm{d}}=0.548 \pm 0.050(\mathrm{stat})_{-0.019}^{+0.023}(\mathrm{syst}) \mathrm{ps}^{-1} .
$$

(To be submitted to Z. Phys.) 


\section{The OPAL Collaboration}

G. Alexander ${ }^{23}$, J. Allison ${ }^{16}$, N. Altekamp ${ }^{5}$, K. Ametewee ${ }^{25}$, K..J. Anderson ${ }^{9}$, S. Anderson ${ }^{12}$, S. Arcelli ${ }^{2}$, S. Asai ${ }^{24}$, D. Axen ${ }^{29}$, G. Azuelos ${ }^{18, a}$, A.H. Ball ${ }^{17}$, E. Barberio ${ }^{26}$, R.J. Barlow ${ }^{16}$, R. Bartoldus ${ }^{3}$, J.R. Batley ${ }^{5}$, G. Beaudoin ${ }^{18}$, J. Bechtluft ${ }^{14}$, C. Beeston ${ }^{16}$, T. Behnke ${ }^{8}$, A.N. Bell ${ }^{1}$, K.W. Bell ${ }^{20}$, G. Bella ${ }^{23}$, S. Bentvelsen ${ }^{8}$, P. Berlich ${ }^{10}$, S. Bethke ${ }^{14}$, O. Biebel ${ }^{14}$, V. Blobel ${ }^{8}$, I.J. Bloodworth ${ }^{1}$,

J.E. Bloomer ${ }^{1}$, P. Bock ${ }^{11}$, H.M. Bosch ${ }^{11}$, M. Boutemeur ${ }^{18}$, B.T. Bouwens ${ }^{12}$, S. Braibant ${ }^{12}$, R.M. Brown ${ }^{20}$, H.J. Burckhart ${ }^{8}$, C. Burgard ${ }^{27}$, R. Bürgin ${ }^{10}$, P. Capiluppi ${ }^{2}$, R.K. Carnegie ${ }^{6}$, A.A. Carter $^{13}$, J.R. Carter ${ }^{5}$, C.Y. Chang ${ }^{17}$, C. Charlesworth ${ }^{6}$, D.G. Charlton ${ }^{1, b}$, D. Chrisman ${ }^{4}$, S.L. Chu ${ }^{4}$, P.E.L. Clarke ${ }^{15}$, I. Cohen ${ }^{23}$, J.E. Conboy ${ }^{15}$, O.C. Cooke ${ }^{16}$, M. Cuffiani ${ }^{2}$, S. Dado ${ }^{22}$, C. Dallapiccola ${ }^{17}$, G.M. Dallavalle ${ }^{2}$, S. De Jong ${ }^{12}$, L.A. del $\mathrm{Pozo}^{8}$, K. Desch ${ }^{3}$, M.S. Dixit ${ }^{7}$, E. do Couto e Silva $^{12}$, M. Doucet ${ }^{18}$, E. Duchovni ${ }^{26}$, G. Duckeck ${ }^{8}$, I.P. Duerdoth ${ }^{16}$, J.E.G. Edwards ${ }^{16}$,

P.G. Estabrooks ${ }^{6}$, H.G. Evans ${ }^{9}$, M. Evans ${ }^{13}$, F. Fabbri ${ }^{2}$, P. Fath ${ }^{11}$, F. Fiedler ${ }^{12}$,

M. Fierro ${ }^{2}$, H.M. Fischer ${ }^{3}$, R. Folman ${ }^{26}$, D.G. Fong ${ }^{17}$, M. Foucher ${ }^{17}$, H. Fukui ${ }^{24}$, A. Fürtjes ${ }^{8}$, P. Gagnon ${ }^{7}$, A. Gaidot ${ }^{21}$, J.W. Gary ${ }^{4}$, J. Gascon ${ }^{18}$, S.M. Gascon-Shotkin ${ }^{17}$, N.I. Geddes ${ }^{20}$, C. Geich-Gimbel ${ }^{3}$, F.X. Gentit ${ }^{21}$, T. Geralis ${ }^{20}$, G. Giacomelli ${ }^{2}$, P. Giacomelli ${ }^{4}$, R. Giacomelli ${ }^{2}$, V. Gibson ${ }^{5}$, W.R. Gibson ${ }^{13}$, D.M. Gingrich ${ }^{30, a}$, J. Goldberg ${ }^{22}$, M.J. Goodrick ${ }^{5}$, W. Gorn ${ }^{4}$, C. Grandi ${ }^{2}$, E. Gross ${ }^{26}$, M. Gruwée C. Hajdu ${ }^{32}$, G.G. Hanson ${ }^{12}$, M. Hansroul ${ }^{8}$, M. Hapke ${ }^{13}$, C.K. Hargrove ${ }^{7}$, P.A. Hart ${ }^{9}$,

C. Hartmann ${ }^{3}$, M. Hauschild ${ }^{8}$, C.M. Hawkes ${ }^{5}$, R. Hawkings ${ }^{8}$, R.J. Hemingway ${ }^{6}$, G. Herten ${ }^{10}$, R.D. Heuer ${ }^{8}$, M.D. Hildreth ${ }^{8}$, J.C.Hill ${ }^{5}$, S.J. Hillier ${ }^{1}$, T. Hilse ${ }^{10}$, J. Hoare ${ }^{5}$,

P.R. Hobson ${ }^{25}$, R.J. Homer ${ }^{1}$, A.K.Honma ${ }^{28, a}$, D. Horváth ${ }^{32, c}$, R. Howard ${ }^{29}$,

R.E. Hughes-Jones ${ }^{16}$, D.E. Hutchcroft ${ }^{5}$, P. Igo-Kemenes ${ }^{11}$, D.C. Imrie ${ }^{25}$,

M.R. Ingram ${ }^{16}$, A. Jawahery ${ }^{17}$, P.W. Jeffreys ${ }^{20}$, H. Jeremie ${ }^{18}$, M. Jimack ${ }^{1}$, A. Joly ${ }^{18}$, C.R. Jones ${ }^{5}$, G. Jones ${ }^{16}$, M. Jones ${ }^{6}$, R.W.L. Jones ${ }^{8}$, U. Jost ${ }^{11}$, P. Jovanovic ${ }^{1}$, T.R. Junk ${ }^{8}$, D. Karlen ${ }^{6}$, K. Kawagoe ${ }^{24}$, T. Kawamoto ${ }^{24}$, R.K. Keeler ${ }^{28}$, R.G. Kellogg ${ }^{17}$,

B.W.Kennedy ${ }^{20}$, B.J. King ${ }^{8}$, J. Kirk ${ }^{29}$, S. Kluth ${ }^{8}$, T. Kobayashi ${ }^{24}$, M. Kobel ${ }^{10}$,

D.S. Koetke ${ }^{6}$, T.P. Kokott ${ }^{3}$, S. Komamiya ${ }^{24}$, R. Kowalewski ${ }^{8}$, T. Kress ${ }^{11}$, P. Krieger ${ }^{6}$, J. von Krogh ${ }^{11}$, P. Kyberd ${ }^{13}$, G.D. Lafferty ${ }^{16}$, H. Lafoux ${ }^{21}$, R. Lahmann ${ }^{17}$, W.P. Lai ${ }^{19}$, D. Lanske ${ }^{14}$, J. Lauber ${ }^{15}$, S.R. Lautenschlager ${ }^{31}$, J.G. Layter ${ }^{4}$, D. Lazic ${ }^{22}$, A.M. Lee ${ }^{31}$,

E. Lefebvre ${ }^{18}$, D. Lellouch ${ }^{26}$, J. Letts ${ }^{2}$, L. Levinson ${ }^{26}$, C. Lewis ${ }^{15}$, S.L. Lloyd ${ }^{13}$,

F.K. Loebinger ${ }^{16}$, G.D. Long ${ }^{17}$, M.J. Losty ${ }^{7}$, J. Ludwig ${ }^{10}$, A. Luig ${ }^{10}$, A. Malik ${ }^{21}$,

M. Mannelli ${ }^{8}$, S. Marcellini ${ }^{2}$, C. Markus ${ }^{3}$, A.J. Martin ${ }^{13}$, J.P. Martin ${ }^{18}$, G. Martinez ${ }^{17}$,

T. Mashimo ${ }^{24}$, W. Matthews ${ }^{25}$, P. Mättig ${ }^{3}$, W.J. McDonald ${ }^{30}$, J. McKenna ${ }^{29}$,

E.A. Mckigney ${ }^{15}$, T.J. McMahon ${ }^{1}$, A.I. McNab ${ }^{13}$, R.A. McPherson ${ }^{8}$, F. Meijers ${ }^{8}$,

S.Menke ${ }^{3}$, F.S. Merritt ${ }^{9}$, H. Mes ${ }^{7}$, J. Meyer $^{27}$, A. Michelini' ${ }^{2}$, G. Mikenberg ${ }^{26}$,

D.J. Miller ${ }^{15}$, R. Mir $^{26}$, W. Mohr ${ }^{10}$, A. Montanari ${ }^{2}$, T. Mori ${ }^{24}$, M. Morii ${ }^{24}$, U. Müller ${ }^{3}$,

H.A. Neal ${ }^{8}$, B. Nellen ${ }^{3}$, B. Nijjhar ${ }^{16}$, R. Nisius ${ }^{8}$, S.W. O'Neale ${ }^{1}$, F.G. Oakham ${ }^{7}$,

F. Odorici ${ }^{2}$, H.O. Ogren ${ }^{12}$, T.Omori ${ }^{24}$, M.J. Oreglia ${ }^{9}$, S. Orito ${ }^{24}$, J. Pálinkás ${ }^{33, d}$,

J.P. Pansart ${ }^{21}$, G. Pásztor ${ }^{32}$, J.R. Pater ${ }^{16}$, G.N.Patrick ${ }^{20}$, M.J. Pearce ${ }^{1}$, S. Petzold ${ }^{27}$, P. Pfeifenschneider ${ }^{14}$, J.E. Pilcher ${ }^{9}$, J. Pinfold ${ }^{30}$, D.E. Plane ${ }^{8}$, P. Poffenberger ${ }^{28}$, 
B. Poli ${ }^{2}$, A. Posthaus ${ }^{3}$, H. Przysiezniak ${ }^{30}$, D.L. Rees ${ }^{1}$, D. Rigby ${ }^{1}$, S.A. Robins ${ }^{13}$, N. Rodning ${ }^{30}$, J.M. Roney ${ }^{28}$, A. Rooke ${ }^{15}$, E. $\operatorname{Ros}^{8}$, A.M. Rossi ${ }^{2}$, M. Rosvick ${ }^{28}$, P. Routenburg ${ }^{30}$, Y. Rozen ${ }^{8}$, K. Runge ${ }^{10}$, O. Runolfsson ${ }^{8}$, U. Ruppe ${ }^{14}$, D.R. Rust ${ }^{12}$, R.Rylko ${ }^{25}$, E.K.G.Sarkisyan ${ }^{23}$, M.Sasaki ${ }^{24}$, C.Sbarra ${ }^{2}$, A.D.Schaile ${ }^{8, e}$, O.Schaile ${ }^{10}$, F.Scharf ${ }^{3}$, P. Scharff-Hansen ${ }^{8}$, P. Schenk ${ }^{4}$, B.Schmitt ${ }^{3}$, S.Schmitt ${ }^{11}$, M.Schröder ${ }^{8}$,

H.C.Schultz-Coulon ${ }^{10}$, M. Schulz ${ }^{8}$, P.Schütz ${ }^{3}$, W.G. Scott ${ }^{20}$, T.G.Shears ${ }^{16}$, B.C.Shen ${ }^{4}$, C.H.Shepherd-Themistocleous ${ }^{27}$, P.Sherwood ${ }^{15}$, G.P.Siroli ${ }^{2}$, A.Sittler ${ }^{27}$, A.Skillman ${ }^{15}$, A.Skuja ${ }^{17}$, A.M.Smith ${ }^{8}$, T.J.Smith ${ }^{28}$, G.A.Snow ${ }^{17}$, R.Sobie ${ }^{28}$, S.Söldner-Rembold ${ }^{10}$, R.W.Springer ${ }^{30}$, M.Sproston ${ }^{20}$, A.Stahl ${ }^{3}$, M.Starks ${ }^{12}$, M.Steiert ${ }^{11}$, K.Stephens ${ }^{16}$, J. Steuerer ${ }^{27}$, B. Stockhausen ${ }^{3}$, D. Strom ${ }^{19}$, F. Strumia ${ }^{8}$, P. Szymanski ${ }^{20}$, R. Tafirout ${ }^{18}$, S.D. Talbot ${ }^{1}$, S. Tanaka ${ }^{24}$, P. Taras ${ }^{18}$, S. Tarem ${ }^{22}$,

M. Tecchio ${ }^{8}$, M. Thiergen ${ }^{10}$, M.A. Thomson ${ }^{8}$, E. von Törne ${ }^{3}$, S. Towers ${ }^{6}$, M. Tscheulin ${ }^{10}$, T. Tsukamoto ${ }^{24}$, E. Tsur ${ }^{23}$, A.S. Turcot ${ }^{9}$, M.F. Turner-Watson ${ }^{8}$, P. Utzat ${ }^{11}$, R. Van Kooten ${ }^{12}$, G. Vasseur ${ }^{21}$, M. Verzocchi ${ }^{10}$, P. Vikas ${ }^{18}$, M. Vincter ${ }^{28}$, E.H. Vokurka ${ }^{16}$, F. Wäckerle ${ }^{10}$, A. Wagner ${ }^{27}$, C.P. Ward ${ }^{5}$, D.R. Ward ${ }^{5}$, J.J. Ward ${ }^{15}$, P.M. Watkins ${ }^{1}$, A.T. Watson ${ }^{1}$, N.K. Watson ${ }^{7}$, P. Weber ${ }^{6}$, P.S. Wells ${ }^{8}$, N. Wermes ${ }^{3}$, J.S. White ${ }^{28}$, B. Wilkens ${ }^{10}$, G.W.Wilson ${ }^{27}$, J.A.Wilson ${ }^{1}$, T. Wlodek ${ }^{26}$, G. Wolf ${ }^{26}$, S. Wotton ${ }^{5}$, T.R. Wyatt ${ }^{16}$, S. Yamashita ${ }^{24}$, G. Yekutieli ${ }^{26}$, V.Zacek ${ }^{18}$,

${ }^{1}$ School of Physics and Space Research, University of Birmingham, Birmingham B15 $2 \mathrm{TT}, \mathrm{UK}$

${ }^{2}$ Dipartimento di Fisica dell' Università di Bologna and INFN, I-40126 Bologna, Italy ${ }^{3}$ Physikalisches Institut, Universität Bonn, D-53115 Bonn, Germany

${ }^{4}$ Department of Physics, University of California, Riverside CA 92521, USA

${ }^{5}$ Cavendish Laboratory, Cambridge CB3 0HE, UK

${ }^{6}$ Ottawa-Carleton Institute for Physics, Department of Physics, Carleton University, Ottawa, Ontario K1S 5B6, Canada

${ }^{7}$ Centre for Research in Particle Physics, Carleton University, Ottawa, Ontario K1S 5B6, Canada

${ }^{8}$ CERN, European Organisation for Particle Physics, CH-1211 Geneva 23, Switzerland ${ }^{9}$ Enrico Fermi Institute and Department of Physics, University of Chicago, Chicago IL 60637, USA

${ }^{10}$ Fakultät für Physik, Albert Ludwigs Universität, D-79104 Freiburg, Germany

${ }^{11}$ Physikalisches Institut, Universität Heidelberg, D-69120 Heidelberg, Germany

${ }^{12}$ Indiana University, Department of Physics, Swain Hall West 117, Bloomington IN 47405, USA

${ }^{13}$ Queen Mary and Westfield College, University of London, London E1 4NS, UK

${ }^{14}$ Technische Hochschule Aachen, III Physikalisches Institut, Sommerfeldstrasse 26-28, D-52056 Aachen, Germany

${ }^{15}$ University College London, London WC1E 6BT, UK

${ }^{16}$ Department of Physics, Schuster Laboratory, The University, Manchester M13 9PL, UK

${ }^{17}$ Department of Physics, University of Maryland, College Park, MD 20742, USA 
${ }^{18}$ Laboratoire de Physique Nucléaire, Université de Montréal, Montréal, Quebec H3C 3.J7, Canada

${ }^{19}$ University of Oregon, Department of Physics, Eugene OR 97403, USA

${ }^{20}$ Rutherford Appleton Laboratory, Chilton, Didcot, Oxfordshire OX11 0QX, UK

${ }^{21}$ CEA, DAPNIA/SPP, CE-Saclay, F-91191 Gif-sur-Yvette, France

${ }^{22}$ Department of Physics, Technion-Israel Institute of Technology, Haifa 32000, Israel

${ }^{23}$ Department of Physics and Astronomy, Tel Aviv University, Tel Aviv 69978, Israel

${ }^{24}$ International Centre for Elementary Particle Physics and Department of Physics, University of Tokyo, Tokyo 113, and Kobe University, Kobe 657, Japan

${ }^{25}$ Brunel University, Uxbridge, Middlesex UB8 3PH, UK

${ }^{26}$ Particle Physics Department, Weizmann Institute of Science, Rehovot 76100, Israel

${ }^{27}$ Universität Hamburg/DESY, II Institut für Experimental Physik, Notkestrasse 85, D-22607 Hamburg, Germany

${ }^{28}$ University of Victoria, Department of Physics, P O Box 3055, Victoria BC V8W 3P6, Canada

${ }^{29}$ University of British Columbia, Department of Physics, Vancouver BC V6T 1Z1, Canada

${ }^{30}$ University of Alberta, Department of Physics, Edmonton AB T6G 2J1, Canada

${ }^{31}$ Duke University, Dept of Physics, Durham, NC 27708-0305, USA

${ }^{32}$ Research Institute for Particle and Nuclear Physics, H-1525 Budapest, P O Box 49, Hungary

${ }^{33}$ Institute of Nuclear Research, H-4001 Debrecen, P O Box 51, Hungary

${ }^{a}$ and at TRIUMF, Vancouver, Canada V6T $2 \mathrm{~A} 3$

${ }^{b}$ and Royal Society University Research Fellow

${ }^{c}$ and Institute of Nuclear Research, Debrecen, Hungary

${ }^{d}$ and Department of Experimental Physics, Lajos Kossuth University, Debrecen, Hungary

${ }^{e}$ and Ludwig-Maximilians-Universität, München, Germany 


\section{Introduction}

Due to second order weak interactions, neutral B mesons oscillate between particle and antiparticle states. The probabilities per unit time that a $B_{q}^{0}(q=d$ or s) produced at time $t=0$ decays as a $\overline{\mathrm{B}}_{\mathrm{q}}^{0}$ or $\mathrm{B}_{\mathrm{q}}^{0}$ at a later time $t$ are:

$$
\begin{aligned}
& P\left(\overline{\mathrm{B}}_{\mathrm{q}}^{0}\right)=\frac{1}{2 \tau} \mathrm{e}^{-t / \tau}\left(1-\cos \left(\Delta m_{\mathrm{q}} t\right)\right) \\
& P\left(\mathrm{~B}_{\mathrm{q}}^{0}\right)=\frac{1}{2 \tau} \mathrm{e}^{-t / \tau}\left(1+\cos \left(\Delta m_{\mathrm{q}} t\right)\right),
\end{aligned}
$$

where $\Delta m_{\mathrm{q}}$, the oscillation frequency, corresponds to the mass difference between the two mass eigenstates and $\tau$ is the $\mathrm{B}_{\mathrm{q}}^{0}$ lifetime. ${ }^{1}$ The contribution of $\Delta \Gamma$, the difference between the total decay widths of the mass eigenstates, to the oscillations is expected to be negligible and is ignored.

This paper reports on two measurements of $\Delta m_{\mathbf{d}}$ using events with $\mathrm{D}^{*+}$ mesons ${ }^{2}$ and leptons. The first measurement uses a sample of reconstructed $\mathrm{D}^{*+}$ mesons with a lepton track in the opposite hemisphere (throughout the rest of this paper, the notation $\mathrm{D}^{*} / \ell$ is used to refer to this sample). The charged $\mathrm{D}^{*}$ tags the decay of a $\mathrm{B}_{\mathrm{d}}^{0}$ meson through the process $\mathrm{B}_{\mathbf{d}}^{0} \rightarrow \mathrm{D}^{*-} \mathrm{X}$. The lepton is used to enrich the b hadron purity of the sample as well as to determine the $\mathrm{b}$ flavor of the $\mathrm{B}_{\mathrm{d}}^{0}$ at production time (i.e. whether it contains $a \mathrm{~b}$ or $\overline{\mathrm{b}})$. This paper supersedes and improves upon a previous measurement of $\Delta m_{\mathrm{d}}$ which also used $\mathrm{D}^{*+}$ mesons opposite leptons [1]. A neural network algorithm is used to improve the performance of the tagging of the $b$ flavor at production. In addition, a new technique is used to measure the proper decay time.

A second and independent analysis has been published using reconstructed charged $\mathrm{D}^{*}$ mesons and lepton tracks in the same hemisphere [2]. This analysis is updated here to include data taken during 1994 . The $\mathrm{B}_{\mathrm{d}}^{0}$ is detected in the channel $\mathrm{B}_{\mathrm{d}}^{0} \rightarrow \mathrm{D}^{*-} \ell^{+} \nu(\mathrm{X})$ and a jet charge technique is used to determine the $\mathrm{b}$ flavor at production (denoted as the $D^{*-} \ell^{+} / Q_{J}$ sample). The analysis technique is unchanged since the previous publication, so only a brief description is given here.

\section{The OPAL Detector and Data Sample}

The OPAL detector has been described elsewhere [3, 4]. Tracking of charged particles is performed by a central detector, consisting of a silicon microvertex detector, a vertex

\footnotetext{
${ }^{1}$ Throughout this paper we use the convention $\hbar=c=1$

${ }^{2}$ Throughout this paper, all references to a particle or decay implicitly include the charge conjugate.
} 
chamber, a jet chamber and $z$-chambers. ${ }^{3}$ The central detector is positioned inside a solenoid, which provides a uniform magnetic field of $0.435 \mathrm{~T}$. The silicon microvertex detector consists of two layers of silicon strip detectors; the inner layer covers a polar angle range of $|\cos \theta|<0.83$ and the outer layer covers $|\cos \theta|<0.77$. This detector provided both $\phi$ - and $z$-coordinates for data taken in 1993 and 1994, but $\phi$-coordinates only for 1991 and 1992. Only $\phi$-coordinate information was used in this analysis. The vertex chamber is a precision drift chamber which covers the range $|\cos \theta|<0.95$. The jet chamber is a large-volume drift chamber, $4 \mathrm{~m}$ long and $3.7 \mathrm{~m}$ in diameter, providing both tracking and $\mathrm{d} E / \mathrm{d} x$ information. The $z$-chambers measure the $z$-coordinate of tracks as they leave the jet chamber in the range $|\cos \theta|<0.72$. The coil is surrounded by a time-of-flight counter array and a lead-glass electromagnetic calorimeter with a presampler. The lead-glass blocks cover the range $|\cos \theta|<0.98$. The magnet return yoke is instrumented with streamer tubes and serves as a hadron calorimeter. Outside the hadron calorimeter are muon chambers, which cover $93 \%$ of the full solid angle.

The data sample used in this paper consists of about 3.5 million hadronic $Z^{0}$ decays collected during the period 1990-1994. The selection of hadronic $Z^{0}$ decays is described in [5]. For the $D^{*} / \ell$ analysis, the data sample is restricted to the period in which the silicon microvertex detector was operational; this amounts to approximately 3 million hadronic $Z^{0}$ decays. Charged tracks and electromagnetic clusters unassociated with any charged track are grouped into jets using the JADE E0 recombination scheme with a $y_{\text {cut }}$ value of 0.04 [6].

Simulated event samples were generated using the JETSET 7.4 Monte Carlo program $[7,8]$, together with a program to simulate the response of the OPAL detector [9]. Production of $L=1 \mathrm{D}$ and $\mathrm{B}$ mesons was included in the simulation [8].

\section{$3 \quad$ The $\mathrm{D}^{*} / \ell$ Analysis}

\subsection{Reconstruction of $\mathrm{D}^{*+}$ and Lepton Candidates}

A sample of events enriched in $B_{d}^{0}$ decays is selected using reconstructed $D^{*+}$ mesons and identified lepton tracks. Each event is divided into two hemispheres which are defined by the plane perpendicular to the $\mathrm{D}^{*+}$ momentum vector. The $\mathrm{D}^{*+}$ candidate and lepton track are required to be in opposite hemispheres. The charge of the $\mathrm{D}^{*}$ meson is used to identify the $b$ quark flavor at the decay time of the parent $\mathrm{B}_{\mathrm{d}}^{0}\left(\mathrm{~B}_{\mathrm{d}}^{0} \rightarrow\right.$ $\left.\mathrm{D}^{*-} \mathrm{X}\right)$. The $\mathrm{D}^{*+}$ candidate is also used to reconstruct the $\mathrm{B}_{\mathrm{d}}^{0}$ decay vertex, from which one obtains an estimate of the decay length of the $\mathrm{B}_{\mathrm{d}}^{0}$. This is combined with an estimate of the relativistic boost of the $B_{d}^{0}$ to give the proper decay time. The charge of the lepton is used to deduce the b quark production flavors in the two hemispheres. By

\footnotetext{
${ }^{3}$ The coordinate system is defined with positive $z$ along the $\mathrm{e}^{-}$beam direction, $\theta$ and $\phi$ being the polar and azimuthal angles. The origin is taken to be the center of the detector.
} 
selecting preferentially leptons from the semileptonic decays of $b$ hadrons, as described in detail later in this section, we ensure a strong correlation between the lepton charge and the charge of the produced $b$ quark in that hemisphere. Assuming $Z^{0} \rightarrow b \bar{b}$ production and taking into account the effects due to time-averaged $\mathrm{B}^{0} \leftrightarrow \overline{\mathrm{B}}^{0}$ mixing, we infer the production flavor of the $b$ hadron in the $\mathrm{D}^{*+}$ hemisphere.

The $\mathrm{D}^{*+}$ mesons are reconstructed through the decay chain

$$
\begin{aligned}
\mathrm{D}^{*+} \rightarrow & \mathrm{D}^{0} \pi^{+} \\
& \rightarrow \mathrm{K}^{-} \pi^{+} .
\end{aligned}
$$

By using the $\mathrm{K}^{-} \pi^{+}$decay mode to reconstruct the $\mathrm{D}^{0}$ we are able to substantially reduce the combinatorial background, relative to other decay modes, which is important for this analysis. Tracks forming the $\mathrm{D}^{*+}$ are required to be contained in the same jet and to pass a set of quality cuts chosen to ensure reliable track reconstruction:

- $\left|d_{0}\right|<0.5 \mathrm{~cm}$

- $\left|z_{0}\right|<20 \mathrm{~cm}$;

- $p_{x y}>0.25 \mathrm{GeV}$; and

- at least 40 hits in the jet chamber,

where $d_{0}$ is the measured distance of closest approach to the nominal $\mathrm{e}^{+} \mathrm{e}^{-}$interaction point in the $x-y$ plane, $z_{0}$ is the $z$ position at that point and $p_{x y}$ is the momentum of the track perpendicular to the beam direction. It is required that at least two of the three tracks in the $\mathrm{D}^{*+}$ candidate have one or more hits in the silicon microvertex detector.

In order to reduce combinatorial background, the tracks forming the $\mathrm{D}^{0}$ candidate are subject to particle identification cuts. For candidate pion tracks, the probability for the measured $\mathrm{d} E / \mathrm{d} x$ value to be consistent with the pion hypothesis is required to be greater than $1 \%$. For candidate kaon tracks, the probability for the kaon hypothesis is required to be greater than $1 \%$ if the measured $\mathrm{d} E / \mathrm{d} x$ is below the expected value. If the measured $\mathrm{d} E / \mathrm{d} x$ is above the expected value, the requirement is tightened to greater than $5 \%$ in order to reduce background from pions.

To further reduce combinatorial background, the $\mathrm{D}^{*+}$ candidates are required to have energy greater than $7 \mathrm{GeV}$. To discriminate against $D^{*+}$ produced in $Z^{0} \rightarrow c \bar{c}$ events, we also require the energy to be less than $30 \mathrm{GeV}$. The decay of the pseudoscalar $\mathrm{D}^{0}$ meson has a flat distribution in $\cos \theta^{*}$, where $\theta^{*}$ is the angle between the $\mathrm{K}^{-}$and the $\mathrm{D}^{0}$ boost direction in the $\mathrm{D}^{0}$ rest frame. The background, however, tends to be concentrated at large $\left|\cos \theta^{*}\right|$. We require $-0.85<\cos \theta^{*}<0.90$. The difference 
between the mass of the $\mathrm{D}^{*+}$ candidate and that of the $\mathrm{D}^{0}$ candidate is required to be in the range $0.144-0.148 \mathrm{GeV}$.

For each $\mathrm{D}^{*+}$ candidate, we require there be an identified electron or muon track whose angle with respect to the $\mathrm{D}^{*+}$ is greater than $90^{\circ}$. Electrons are identified using an artificial neural network [10] and muons are identified as described in [11]. Both types of lepton are required to have momentum greater than $2 \mathrm{GeV}$ and transverse momentum with respect to the axis of the jet containing the lepton greater than 0.5 $\mathrm{GeV}$. The lepton track is subject to the same track quality cuts listed above for tracks forming the $\mathrm{D}^{*+}$ candidate. The sample is enriched in direct $\mathrm{b} \rightarrow \ell$ decays by using the output of a neural network based on kinematic variables. This neural network is the same as in [12], except that an additional jet charge variable is included to enhance the accuracy of the flavor tagging. The neural network is of the feed-forward type and uses the following four input quantities:

- the lepton momentum $p$;

- the lepton momentum transverse to the direction of the jet containing the lepton, $p_{t}$, where the jet direction is computed including the lepton momentum;

- the isolation of the lepton track from other tracks and electromagnetic clusters in the jet containing the lepton (see [12] for a more detailed description);

- the product of the lepton charge and the jet charge of the jet containing the lepton, defined as $q_{\ell} \cdot Q$, where:

$$
Q=\sum_{i=1}^{n} q_{i} \cdot\left(\frac{p_{l, i}}{E_{\text {beam }}}\right)
$$

where the sum runs over all the tracks in the jet, excluding the lepton, $q_{\ell}$ is the charge of the lepton, $q_{i}$ is the charge of track $i, p_{l, i}$ is the momentum of track $i$ parallel to the jet axis and $E_{\text {beam }}$ is the beam energy.

The network is trained to distinguish between leptons with the same sign of charge as the parent $b$ quark and those with the opposite sign. Leptons of opposite sign come from processes such as $\mathrm{b} \rightarrow \mathrm{c} \rightarrow \ell$ and $\mathrm{B}^{0} \rightarrow \overline{\mathrm{B}}^{0} \rightarrow \ell$. We cut on the neural network output such that the mistag rate (the probability of inferring incorrectly the production flavor of the $\mathrm{B}$ meson) predicted by the Monte Carlo sample is $0.219 \pm 0.026$, where the error is due predominantly to the statistics of the Monte Carlo sample. This cut choice has approximately the same efficiency as for cutting on $p>3 \mathrm{GeV}$ and $p_{t}>0.75 \mathrm{GeV}$, but in that case the mistag rate is predicted to be $0.273 \pm 0.029$. In calculating the mistag rates, we use the most recent OPAL measurement for the average mixing parameter, $\bar{\chi}=0.1107 \pm 0.0062 \pm 0.0055[10]$, where $\bar{\chi}$ is the probability that a produced $b$ hadron decays as its antiparticle. The neural network is clearly a valuable aid in primary quark charge tagging. 


\subsection{Reconstruction of $\mathrm{B}_{\mathrm{d}}^{0}$ Proper Decay Time}

The $\mathrm{B}_{\mathbf{d}}^{0}$ proper decay time, $t$, can be expressed as $t=L /(\beta \gamma)$, where $L$ is the decay length of the $\mathrm{B}_{\mathrm{d}}^{0}$ and $\beta \gamma$ is the Lorentz boost of the $\mathrm{B}_{\mathrm{d}}^{0}$. For the previous measurement of $\Delta m_{\mathrm{d}}$ using $\mathrm{D}^{*+}$ mesons and leptons in opposite hemispheres, neither the $\mathrm{B}_{\mathrm{d}}^{0}$ decay length nor its boost was estimated [1]. Instead, the $\mathrm{D}^{0}$ decay vertex was used, which allowed for a measurement of the sum of the decay lengths of the $D^{0}$ and the $B_{d}^{0}$ mesons. Since the energy of the $\mathrm{B}_{\mathrm{d}}^{0}$ meson was not estimated, we performed a fit to the decay length distribution, assuming a momentum distribution of $\mathrm{B}$ mesons according to a given fragmentation function. This analysis improves upon the previous one by estimating both the $\mathrm{B}_{\mathrm{d}}^{0}$ decay length and its boost, on an event-by-event basis. This section describes the method used to separately estimate the decay length and the boost of the assumed parent $\mathrm{B}_{\mathrm{d}}^{0}$ meson.

The primary event vertex is reconstructed using the charged tracks in the event, excluding those used in the $\mathrm{D}^{*+}$ candidate as well as the lepton track, along with knowledge of the average position and effective spread of the $\mathrm{e}^{+} \mathrm{e}^{-}$collision point. In this process, tracks that are significantly separated from the vertex position are excluded from the final vertex reconstruction.

The $\mathrm{D}^{0}$ vertex is formed from a fit using the three tracks forming the $\mathrm{D}^{*+}$ candidate. The slow pion track from the $\mathrm{D}^{*+}$ decay is included in the vertex fit since its direction follows closely that of the $D^{0}$ and so can be used to constrain the $D^{0}$ direction. The parameters and error matrices of the tracks which form the $\mathrm{D}^{*+}$ candidate are used to form a $\mathrm{D}^{*+}$ pseudo-track. A vertex algorithm is used to combine the candidate $\mathrm{D}^{*+}$ pseudo-track with other tracks in the same jet which are deemed likely to be products of the $\mathrm{B}_{\mathrm{d}}^{0}$ decay. In order to be considered by the vertex algorithm, each track must pass the following cuts:

- It must be precisely measured by either the silicon microvertex detector or the vertex drift chamber.

- The intersection of the track with the $\mathrm{D}^{*+}$ trajectory, defined as the line through the $\mathrm{D}^{0}$ decay vertex in the direction of the total reconstructed momentum of the $\mathrm{D}^{*+}$ decay products, is required to be consistent with coming from the decay of a b hadron. Specifically, the distance, $L_{D}$, between this intersection and the $\mathrm{D}^{0}$ decay vertex must satisfy $L_{D} / \sigma_{D}>-2.5$, where $L_{D}$ is signed negative only if the point of intersection lies further from the primary vertex than the $\mathrm{D}^{0}$ decay vertex, and $\sigma_{D}$ is the uncertainty on $L_{D}$. The requirement $t_{D} / \tau_{D}<5$ is also imposed, where $t_{D}=L_{D} /(\beta \gamma)_{D}$ is the measured proper decay time of the $\mathrm{D}^{0}$ and $\tau_{D}$ is the $\mathrm{D}^{0}$ lifetime.

- The probability of the track to come from b hadron decay compared to fragmentation must be greater than $10 \%$. This probability is based on the track momentum and its angle with respect to the $\mathrm{D}^{*+}$ direction, which are correlated. 
The $b$ hadron decay products tend to have a harder momentum spectrum and be more collimated about the $\mathrm{D}^{*+}$ direction than tracks from fragmentation. A Monte Carlo sample was used to parametrize the probability with respect to this angle for different track momentum ranges.

Tracks passing these criteria are then ordered according to their significance of separation with respect to the primary vertex. If only a single track is left, its intersection with the $\mathrm{D}^{*+}$ pseudo-track is considered to be the $\mathrm{b}$ hadron decay vertex. If two tracks remain, a common vertex is formed between them and the $D^{*+}$ pseudo-track. The vertex is rejected if the $\chi^{2}$ probability of the fit is less than $1 \%$. If more than two tracks remain, a seed vertex is formed using the two tracks for which the vertex with the $\mathrm{D}^{*+}$ pseudo-track has the highest $\chi^{2}$ probability. The remaining tracks are combined, oneby-one, with the seed vertex to form a candidate vertex. If the addition of any given track to the vertex causes the $\chi^{2}$ probability to drop below $1 \%$, the track is removed from the vertex and the next track is considered. The vertex finding is performed in the $x-y$ plane.

The decay length in the $x-y$ plane is taken as the projection along the $\mathrm{D}^{*+}$ direction of the vector between the primary vertex and the $b$ hadron decay vertex. The decay length is converted into three dimensions using the polar angle of the $\mathrm{D}^{*+}$ vector. Candidate vertices with negative decay lengths more than three standard deviations from the primary vertex are rejected. The efficiency for reconstructing a vertex, given a $\mathrm{D}^{*+}$ candidate, is approximately $70 \%$ and is independent of the decay length.

The energy of the $\mathrm{b}$ hadron is estimated using a method identical to that used in a previous $\Delta m_{\mathbf{d}}$ analysis of OPAL [12], with the exception that this analysis tags b hadrons in their decays to $\mathrm{D}^{*+}$ mesons while the previous one used inclusive semileptonic decays. The energy of the jet containing the $\mathrm{D}^{*+}$ is reconstructed, using the $\mathrm{Z}^{0}$ mass to constrain the event kinematics, and then the estimated contribution from fragmentation particles is subtracted. The proper decay time, $t$, is then formed from the decay length $L$ and the boost. The uncertainty on the proper decay time, $\sigma_{t}$, is calculated from the separately estimated uncertainties on the decay length, $\sigma_{L}$, and the b hadron energy, $\sigma_{E_{B}}$ :

$$
\left(\frac{\sigma_{t}}{t}\right)^{2}=\left(\frac{\sigma_{L}}{L}\right)^{2}+\left(\frac{\sigma_{E_{B}}}{E_{B}}\right)^{2}
$$

The decay length uncertainty is calculated from the errors on the primary vertex and the error matrices of the $\mathrm{D}^{*+}$ and the tracks assigned to the $\mathrm{b}$ hadron vertex. The average uncertainty on the energy measurement is estimated to be $5 \mathrm{GeV}$, based on Monte Carlo studies, and we fix $\sigma_{E_{B}}$ to this value. Figure 1 shows the distribution of $\sigma_{t}$ for events passing all of the cuts described above, for both data and Monte Carlo events. To reject bogus and poorly reconstructed vertices, we require $|t|<12$ ps and $\sigma_{t}<1.2$ ps.

The distribution of the reconstructed proper decay time versus the true proper decay time is shown in Figure 2 for Monte Carlo simulated $\mathrm{B}_{\mathrm{d}}^{0} \rightarrow \mathrm{D}^{*-} \mathrm{X}$ decays. The 
assignment of tracks to the $b$ hadron vertex is imperfect, which results in tails in the deviation of the measured time from the true time and causes a fraction of the vertices to be misreconstructed near the primary vertex even when the true decay length is large. These effects are evident in Figure 2 and in Figure 3, which shows the deviation of the reconstructed time from the true time, $t-t^{\prime}$, as well as the normalized quantity $\left(t-t^{\prime}\right) / \sigma_{t}$, where $t^{\prime}$ is the true proper decay time.

\subsection{The Signal and Background Fractions}

The $\mathrm{K}^{-} \pi^{+}$invariant mass distribution, after applying all of the cuts described in the previous sections, is displayed in Figure 4. The signal is parametrized by a Gaussian. The background is parametrized by a second-order polynomial and a Gaussian near $1.61 \mathrm{GeV}$ to account for an enhancement arising from partially reconstructed decays, particularly $\mathrm{D}^{0} \rightarrow \mathrm{K}^{-} \pi^{+} \pi^{0}$. Because the signal-to-background ratio for these partially reconstructed $\mathrm{D}^{0}$ decays is poor, they are not used in this analysis. In the signal region, defined to be 1.791-1.925 GeV, there is a total of 348 events. The combinatorial background is determined from the fit to the $\mathrm{D}^{0}$ invariant mass distribution. After subtracting the estimated $95 \pm 11$ combinatorial background events, the number of $D^{0}$ decays is found to be $253 \pm 19$.

In addition to $\mathrm{B}_{\mathrm{d}}^{0}$ decays, the following background processes contribute to the selected events:

1. the production of $\mathrm{D}^{*+}$ mesons in $\mathrm{Z}^{0} \rightarrow \mathrm{c} \overline{\mathrm{c}}$ events,

2. the decays of $\mathrm{B}_{\mathrm{s}}^{0}$ mesons into states containing a $\mathrm{D}^{*+}$,

3. the decays of $\mathrm{B}^{-}$mesons into states containing a $\mathrm{D}^{*+}$,

4. combinatorial background.

We use the prediction from the Monte Carlo simulation for the number of events from background sources 1 and 2. The inclusive branching ratios for $\mathrm{B}_{\mathrm{d}}^{0}$ and $\mathrm{B}^{-}$mesons decaying into $\mathrm{D}^{*+}$ mesons have not been measured. The fraction of $\mathrm{D}^{*+}$ from semileptonic decays of $\mathrm{B}^{-}$relative to those of $\mathrm{B}_{\mathrm{d}}^{0}$ has been estimated, however, to be $(16 \pm 9) \%$ [13]. One does not expect a large difference for hadronic B meson decays. Thus, we assume this value, but allow the fraction to vary in the fit. The number of $B_{d}^{0}$ decays in the sample is given by the difference between the total number of selected events and the sum of the background contributions.

In order to measure the frequency of $\mathrm{B}_{\mathrm{d}}^{0}$ oscillations, we distinguish between like-sign $\left(\mathrm{D}^{*+} / \ell^{+}\right)$and unlike-sign $\left(\mathrm{D}^{*+} / \ell^{-}\right)$combinations. The $\mathrm{D}^{*+}$ yield is $161 \pm 15$ for likesign combinations and $92 \pm 12$ for unlike-sign combinations. For perfect production and decay flavor tagging, the like-sign sample would contain all of the unmixed $B_{d}^{0}$ 
decays, all of the $\mathrm{B}^{-}$decays and none of the $\mathrm{Z}^{0} \rightarrow c \bar{c}$ events. Likewise, the unlikesign sample would contain all of the mixed $\mathrm{B}_{\mathrm{d}}^{0}$ decays, none of the $\mathrm{B}^{-}$decays and all of the $\mathrm{Z}^{0} \rightarrow c \bar{c}$ events. Assuming the $\mathrm{B}_{\mathrm{s}}^{0}$ mixing is close to maximal, the $\mathrm{B}_{\mathrm{s}}^{0}$ decays would equally populate the like and unlike-sign samples. For $b \bar{b}$ events, the tagging of the production flavor is imperfect due to the presence of leptons from cascade decays $(\mathrm{b} \rightarrow \mathrm{c} \rightarrow \ell)$, leptons from $\mathrm{B}$ mesons which have mixed before decaying $\left(\mathrm{B}^{0} \rightarrow \overline{\mathrm{B}}^{0} \rightarrow \ell\right)$ and fake lepton tracks. For $\mathrm{Z}^{0} \rightarrow c \overline{\mathrm{c}}$ events, the only contribution is from fake leptons. We define the $B_{d}^{0}$ mistag rate, $\eta_{\mathrm{b}}$, to be the probability that an unmixed $\mathrm{B}_{\mathrm{d}}^{0}$ decay with a correctly reconstructed $\mathrm{D}^{*+}$ will fall in the unlike-sign sample or a mixed $\mathrm{B}_{\mathrm{d}}^{0}$ decay in the like-sign sample. Likewise, the charm mistag rate, $\eta_{c}$, is defined to be the probability that a $\mathrm{D}^{*+}$ from the process $\mathrm{Z}^{0} \rightarrow \mathrm{c} \overline{\mathrm{c}}$ will fall in the like-sign sample. For the $\mathrm{B}^{-}$meson case, we allow for a correction to the mistag rate, $\eta_{\mathrm{b}}$, arising from decays of the type $\mathrm{B}^{-} \rightarrow \mathrm{D}^{*-} \mathrm{X}$, which is suppressed relative to $\mathrm{B}^{-} \rightarrow \mathrm{D}^{*+} \mathrm{X}$. The former process introduces an effective decay flavor mistag which increases the overall mistag rate. For the $B_{d}^{0}$ case, decays of the type $B_{d}^{0} \rightarrow D^{*+} X$ are expected to have a negligible rate relative to the process $B_{d}^{0} \rightarrow D^{*-} X$. We define the quantity $\delta^{+}$to be the ratio of the $\mathrm{B}^{-}$mistag rate to the $\mathrm{B}_{\mathrm{d}}^{0}$ mistag rate. From Monte Carlo samples, we estimate that the ratio of mistag rates for $\mathrm{B}_{\mathrm{s}}^{0}$ decays and $\mathrm{B}_{\mathrm{d}}^{0}$ decays is $1.25 \pm 0.22$.

Table 1 lists the estimated number of events in the selected sample for each source listed above, as well as the mistag values. The errors reflect the uncertainties in branching fractions and production rates used as input to the Monte Carlo simulation, as well as the statistical errors from efficiency calculations.

\begin{tabular}{|c|c|}
\hline Quantity & Value \\
\hline Number of $B_{d}^{0}$ decays & $167 \pm 25$ \\
\hline Number of $\mathrm{B}^{-}$decays & $29 \pm 16$ \\
\hline Number of $\mathrm{B}_{\mathrm{s}}^{0}$ decays & $17 \pm 8$ \\
\hline Number of $c \bar{c}$ events & $40 \pm 13$ \\
\hline Amount of combinatorial bkgd & $95 \pm 11$ \\
\hline$\eta_{\mathrm{b}}$ & $0.219 \pm 0.026$ \\
\hline$\delta^{+}$ & $1.21 \pm 0.20$ \\
\hline$\eta_{c}$ & $0.225 \pm 0.044$ \\
\hline
\end{tabular}

Table 1: Summary of sample composition and mistag values for selected $\mathrm{D}^{*} / \ell$ events. $\eta_{\mathrm{b}}$ is the mistag value for $\mathrm{B}_{\mathrm{d}}^{0}$ decays, $\delta^{+}$is the ratio of the $\mathrm{B}^{-}$mistag rate to the $\mathrm{B}_{\mathrm{d}}^{0}$ mistag rate and $\eta_{c}$ is the charm mistag rate.

\subsection{The Maximum Likelihood Fit}

An unbinned maximum likelihood fit is used for estimating $\Delta m_{\mathrm{d}}$. The likelihood is the product of the individual likelihoods, $\mathcal{L}_{i}$, for each event $i$. It takes into account 
the underlying physics principles, the effects due to detector resolution and the limited knowledge of the input parameters. The likelihood for any given event is a function of the independent variables $t_{i}$, the measured proper decay time; $\sigma_{t}$, the calculated error on the proper decay time; and $\rho=q_{\mathrm{D}^{*}} \cdot q_{\ell}$, the product of the $\mathrm{D}^{*}$ and lepton charges.

For each event, the full likelihood is the sum of terms describing the signal and background contributions:

$$
\begin{aligned}
\mathcal{L}_{i} & =\mathcal{L}_{i}^{\mathrm{D}^{*}}\left(1-f_{i}^{c o m b}\right)+\mathcal{L}_{i}^{c o m b} f_{i}^{c o m b}, \\
\mathcal{L}_{i}^{\mathrm{D}^{*}} & =\mathcal{L}_{i}^{\mathrm{B}_{\mathrm{d}}} f^{\mathrm{B}_{\mathrm{d}}}+\mathcal{L}_{i}^{\mathrm{B}^{-}} f^{\mathrm{B}^{-}}+\mathcal{L}_{i}^{\mathrm{B}_{3}} f^{\mathrm{B}_{3}}+\mathcal{L}_{i}^{c} f^{c} .
\end{aligned}
$$

The various quantities are defined in the following way:

- $\mathcal{L}_{i}^{c o m b}$ is the probability density function for the combinatorial background.

- $\mathcal{L}_{i}^{\mathrm{B}_{\mathrm{d}}}, \mathcal{L}_{i}^{\mathrm{B}^{-}}$and $\mathcal{L}_{i}^{\mathrm{B}_{3}}$ are the probability density functions for $\mathrm{B}_{\mathrm{d}}^{0}, \mathrm{~B}^{-}$, and $\mathrm{B}_{\mathrm{s}}^{0}$ decays, respectively.

- $\mathcal{L}_{i}^{c}$ is the probability density function for $Z^{0} \rightarrow c \bar{c}$ events.

- $f_{i}^{c o m b}$ is the time-integrated probability that a $\mathrm{D}^{*+}$ candidate is from combinatorial background. It is a function of the reconstructed $\mathrm{K}^{-} \pi^{+}$mass and is calculated from the fit to the mass distribution.

- $f^{\mathrm{B}_{\mathrm{d}}}, f^{\mathrm{B}^{-}}, f^{\mathrm{B}_{\mathrm{s}}}$ and $f^{\mathrm{c}}$ are the fractions of $\mathrm{D}^{*+}$ signal events coming from $\mathrm{B}_{\mathrm{d}}^{0}, \mathrm{~B}^{-}$, $\mathrm{B}_{\mathrm{s}}^{0}$ and $\mathrm{Z}^{0} \rightarrow \mathrm{c} \overline{\mathrm{c}}$, respectively. These fractions sum to one.

The normalization of each probability density, $\mathcal{L}_{i}^{j}\left(j=\mathrm{B}_{\mathrm{d}}, \mathrm{B}^{-}, \mathrm{B}_{\mathrm{s}}^{0}\right.$, c or comb), includes the sum over $\rho$, the product of the $\mathrm{D}^{*}$ and lepton charges:

$$
\sum_{\rho} \int_{-\infty}^{\infty} \mathcal{L}_{i}^{j}(t) \mathrm{d} t=1
$$

Each probability density function is obtained from the convolution of a physics function $\mathcal{P}^{j}\left(t^{\prime}\right)$ with a resolution function $\mathcal{R}^{j}\left(t, t^{\prime}, \sigma_{t}\right)$. The $\mathrm{B}_{\mathrm{d}}^{0}$ physics function is

$$
\mathcal{P}^{\mathrm{B}_{\mathrm{d}}}=\frac{e^{-t^{\prime} / \tau_{\mathrm{B}_{\mathrm{d}}}}}{\tau_{\mathrm{B}_{\mathrm{d}}}} \cdot \frac{\eta_{\mathrm{b}}\left(1-\rho \cos \left(\Delta m_{\mathrm{d}} t^{\prime}\right)\right)+\left(1-\eta_{\mathrm{b}}\right)\left(1+\rho \cos \left(\Delta m_{\mathrm{d}} t^{\prime}\right)\right)}{2},
$$

where $\tau_{\mathrm{B}_{\mathrm{d}}}$ is the $\mathrm{B}_{\mathrm{d}}^{0}$ lifetime.

The $\mathrm{B}_{\mathrm{s}}^{0}$ physics function has the same form as that for $\mathrm{B}_{\mathrm{d}}^{0}$, with the appropriate parameters for $\mathrm{B}_{\mathrm{s}}^{0}$ decays $\left(\Delta m_{\mathrm{s}}, \tau_{\mathrm{B}_{\mathrm{s}}}\right.$ and the $\mathrm{B}_{\mathrm{s}}^{0}$ mistag rate). The $\mathrm{B}^{-}$physics function also has the same form, but has an oscillation frequency of zero. 
For the combinatorial background and $Z^{0} \rightarrow c \bar{c}$ terms, the physics function is empirically chosen to be a single exponential. This component with small non-zero lifetime is due to tracks from b or c hadron decays being included in the reconstructed vertex. The lifetime parameter is obtained from fitting Monte Carlo simulated events, for the case of $Z^{0} \rightarrow c \bar{c}$ events, or the $D^{0}$ mass sideband defined as $\mathrm{K}^{-} \pi^{+}$invariant mass between 2.0 and $2.4 \mathrm{GeV}$, for the case of combinatorial background (assuming resolution functions as described below).

In all cases, the resolution function consists of two Gaussians centered at $t=t^{\prime}$, to model resolution effects, and a third Gaussian centered at $t=0$, describing the fraction of events which are misreconstructed near the primary vertex, independent of the proper decay time $t^{\prime}$ in the physics functions. The Monte Carlo simulated events are used to fit for the fractions of the three Gaussians and their widths. For the combinatorial background, we use, instead, events in the $\mathrm{D}^{0}$ sideband region.

The resolution function for $\mathrm{B}_{\mathrm{d}}^{0}$ decays is displayed in Figure 3. Figure 5 shows the reconstructed proper decay time distributions for the signal and background components, with the parametrizations used in the fit overlayed.

\subsection{Measurement of the Frequency of $B_{d}^{0}$ Oscillation}

The total likelihood for the selected sample is

$$
\mathcal{L}\left(p_{1}, p_{2}, \ldots p_{m}\right)=\prod_{i=1}^{n} \mathcal{L}_{i}\left(t_{i}, \sigma_{t_{i}} \mid p_{1}, p_{2}, \ldots p_{m}\right),
$$

where $p_{1}, p_{2}, \ldots p_{m}$ are the parameters whose values are determined from the maximum likelihood fit. The parameter $\Delta m_{\mathrm{d}}$ is allowed to vary freely in the fit, while the others are given Gaussian constraints. This is the same procedure as used in a previous paper [12]. The parameters which are allowed to vary, along with their constraints, are given in Table 2. Also shown are the final values for the parameters at the point of maximum likelihood. The fitted value of $\Delta m_{\mathrm{d}}$ is $0.567 \pm 0.093 \mathrm{ps}^{-1}$, where the error includes the systematic error arising from variation of the other parameters in the fit. The fractions are defined as follows: $\mathrm{F}^{c}=f^{c}, \mathrm{~F}^{\mathrm{B}_{\mathrm{s}}}=f^{\mathrm{B}_{\mathrm{s}}} /\left(f^{\mathrm{B}_{\mathrm{s}}}+f^{\mathrm{B}_{\mathrm{d}}}+f^{\mathrm{B}^{-}}\right)$ and $\mathrm{F}^{\mathrm{B}^{-}}=f^{\mathrm{B}^{-}} /\left(f^{\mathrm{B}_{\mathrm{d}}}+f^{\mathrm{B}^{-}}\right)$. The fraction of $\mathrm{B}_{\mathrm{d}}^{0}$ decays, $\mathrm{F}^{\mathrm{B}_{\mathrm{d}}}$, is not an independent parameter in the fit, but is constrained by the condition $\mathrm{F}^{\mathrm{B}_{\mathrm{d}}}=f^{\mathrm{B}_{\mathrm{d}}}=1-f^{\mathrm{B}^{-}}-f^{\mathrm{B}_{\mathrm{B}}}-f^{\mathrm{c}}$. Using the nominal input values for the other fractions, $\mathrm{F}^{\mathrm{B}_{\mathrm{d}}}=0.651 \pm 0.098$. Using the output values from the fit, $\mathrm{F}^{\mathrm{B}_{\mathrm{d}}}=0.81_{-0.11}^{+0.05}$.

This fitting procedure naturally incorporates the principal uncertainties, which arise from the limited knowledge of the parameters listed in Table 2 . The value of $-\Delta \log \mathcal{L}$ as a function of $\Delta m_{\mathrm{d}}$ is shown in Figure 6 , where $\Delta \log \mathcal{L}$ denotes the difference in $\log \mathcal{L}$ relative to its maximum value. Figure 7 shows the observed proper decay time distribution for the selected events, with the curve resulting from the fit overlaid. Figure 8 shows the ratio of like-sign to total events as a function of proper decay time. 


\begin{tabular}{|c|c|c|}
\hline Parameter & Input & Output from fit \\
\hline$\Delta m_{\mathrm{d}}$ & & $0.567 \pm 0.093 \mathrm{ps}^{-1}$ \\
$\mathrm{~F}^{\mathrm{B}^{-}}$ & $0.16 \pm 0.09$ & $0.02_{-0.02}^{+0.13}$ \\
$\mathrm{~F}^{\mathrm{B}_{\mathrm{B}}}$ & $0.080 \pm 0.040$ & $0.043 \pm 0.039$ \\
$\mathrm{~F}^{\mathrm{c}}$ & $0.159 \pm 0.050$ & $0.141 \pm 0.033$ \\
$\eta_{\mathrm{b}}$ & $0.219 \pm 0.026$ & $0.204 \pm 0.024$ \\
$\delta^{+}$ & $1.21 \pm 0.20$ & $1.21 \pm 0.19$ \\
$\eta_{\mathrm{c}}$ & $0.225 \pm 0.044$ & $0.233 \pm 0.048$ \\
\hline
\end{tabular}

Table 2: The parameters in the fit, their constraints and their final values after maximizing $\log \mathcal{L}$. The errors in the last column are those returned from the fit.

\subsection{Study of Systematic Uncertainties}

As discussed above, the main uncertainties are included in the fit. There remain, however, other less important sources of systematic uncertainty which are evaluated separately. This is accomplished by varying each source of systematic error by one standard deviation, repeating the fit and noting the change in $\Delta m_{\mathrm{d}}$. These sources are listed below:

- The $\mathbf{B}_{\mathbf{d}}^{\mathbf{0}}, \mathbf{B}^{-}$and $\mathbf{B}_{\mathrm{s}}^{\mathbf{0}}$ lifetimes: For the fit, we use $\tau_{\mathrm{B}_{\mathrm{d}}}=1.56 \pm 0.06 \mathrm{ps}$ [14], $\tau_{\mathrm{B}^{-}} / \tau_{\mathrm{B}_{\mathrm{d}}}=1.03 \pm 0.06$ [14] and $\tau_{\mathrm{B}_{\mathrm{s}}}=1.61 \pm 0.10$ [15]. The variation of $\tau_{\mathrm{B}_{\mathrm{d}}}$ results in a change in $\Delta m_{\mathrm{d}}$ of $\mp 0.002 \mathrm{ps}^{-1}$. The uncertainty in the lifetime difference between $\mathrm{B}_{\mathrm{d}}^{0}$ and $\mathrm{B}^{-}$results in a change in $\Delta m_{\mathrm{d}}$ of $\pm 0.006 \mathrm{ps}^{-1}$. The uncertainty in the $\mathrm{B}_{\mathrm{s}}^{0}$ lifetime contributes a systematic error of $\pm 0.002 \mathrm{ps}^{-1}$. The $\mathrm{B}_{\mathrm{d}}^{0}$ lifetime is held fixed when varying $\tau_{\mathrm{B}^{-}}$and $\tau_{\mathrm{B}_{\mathrm{B}}}$.

- The combinatorial background fraction: The overall fraction of combinatorial background is measured to be $0.273 \pm 0.026$. Varying this fraction within its error results in a change in $\Delta m_{\mathrm{d}}$ of $\mp 0.004 \mathrm{ps}^{-1}$.

- The charge correlation of the combinatorial background: By fitting the $\mathrm{D}^{0}$ invariant mass distributions of the like-sign and unlike-sign samples separately, we determine the ratio of like-sign to total background to be $0.40 \pm 0.05$. Variation of this quantity within its errors results in a systematic variation of $\pm 0.003 \mathrm{ps}^{-1}$ in $\Delta m_{\mathrm{d}}$.

- The proper decay time resolution: To obtain the uncertainty due to imprecise knowledge of the detector resolution, we recalculate the resolution functions after improving and degrading the nominal tracking resolution in the Monte Carlo simulation by $20 \%$. The observed change in the fitted value of $\Delta m_{\mathrm{d}}$ is 
$\pm 0.002 \mathrm{ps}^{-1}$. As an additional check, the fit has been repeated using alternative parametrizations of the $\mathrm{B}$ meson resolution function. We considered a parametrization which allows for biases in the reconstructed proper decay time as well as one which has a bend in the proper decay time distribution at $t=5 \mathrm{ps}$. The motivation for this second parametrization is to better model the observed reconstructed proper time distributions in the region above 5 ps (see Figure 5 ). The observed variation in $\Delta m_{\mathrm{d}}$ using other resolution functions is $\pm 0.002 \mathrm{ps}^{-1}$. Summing in quadrature these two quantities, we estimate a total systematic error of $\pm 0.003 \mathrm{ps}^{-1}$ arising from uncertainties in the proper decay time resolution.

- The $\mathbf{B}_{\mathrm{s}}^{0}$ oscillation frequency: For the fit we use a nominal value of $\Delta m_{\mathrm{s}}=20$ $\mathrm{ps}^{-1}$. Varying this quantity in the range $2 \mathrm{ps}^{-1}$ to $40 \mathrm{ps}^{-1}$ results in a negligible change in the fitted value of $\Delta m_{\mathrm{d}}$, and no additional systematic error is assigned.

The contributions to the systematic uncertainty from all sources considered are summarized in Table 3. The uncertainties from the parameters in Table 2 are calculated by individually varying the central value of each constraint by its error and repeating the full 6 parameter fit. Since the error returned from the fit already includes these uncertainties, they are subtracted, in quadrature, from the fit error $\left( \pm 0.093 \mathrm{ps}^{-1}\right)$ in order to obtain an estimate of the pure statistical uncertainty. We find a statistical uncertainty of $\pm 0.089 \mathrm{ps}^{-1}$.

This measurement is checked by repeating the fit using different cuts and techniques. By cutting harder on the lepton neural net output, one can obtain a sample with greater $\mathrm{B}_{\mathrm{d}}^{0}$ purity and smaller mistag rate, at the cost of decreased statistics. We have repeated the fit choosing a neural net cut that yields a predicted mistag rate of $0.163 \pm 0.032$ and a reduction of $\mathrm{F}^{c}$ to $0.039_{-0.039}^{+0.050}$, along with a $50 \%$ loss in reconstructed $\mathrm{D}^{*+}$ mesons. Using the same fitting technique described previously, we obtain $\Delta m_{\mathbf{d}}=0.52 \pm 0.11$ $\mathrm{ps}^{-1}$. We have also repeated the fit using more traditional lepton $p$ and $p_{t}$ cuts in place of the neural net cut. For $p>3 \mathrm{GeV}$ and $p_{t}>0.75 \mathrm{GeV}$, the mistag rate is predicted to be $0.273 \pm 0.029$ and the efficiency is approximately the same as for the nominal neural net cut. In this case, we measure $\Delta m_{\mathrm{d}}=0.54 \pm 0.10 \mathrm{ps}^{-1}$. In both cases, the measured value of $\Delta m_{\mathrm{d}}$ is consistent with the result using the nominal choice of cuts, within the independent statistical errors, and no additional systematic error is assigned.

Studies were performed on Monte Carlo samples which contained the same signal and background statistics as the selected data sample, but with the fraction of $\mathrm{B}^{-}$ decays, $\mathrm{F}^{\mathrm{B}^{-}}$, varied within the range 0 to 0.30 . In all cases, the fitted values of $\Delta m_{\mathrm{d}}$ and $\mathrm{F}^{\mathrm{B}^{-}}$were consistent with the generated values.

We also measure the average $b$ hadron lifetime as a check of the proper decay time reconstruction and fit method. We measure a lifetime of $\tau_{\mathrm{b}}=1.44 \pm 0.16 \mathrm{ps}$, where $\tau_{\mathrm{b}}$ is the average lifetime for the mixture of $\mathrm{b}$ hadrons in our sample. This value is consistent with the lifetimes we assumed in the $\Delta m_{\mathrm{d}}$ fit. 


\begin{tabular}{|c|c|}
\hline Source of error & Error on $\Delta m_{\mathrm{d}}\left(\mathrm{ps}^{-1}\right)$ \\
\hline $\mathrm{F}^{\mathrm{B}^{-}}$ & +0.017 \\
$\mathrm{~F}^{\mathrm{B}_{\mathrm{B}}}$ & -0.002 \\
$\mathrm{~F}^{\mathrm{c}}$ & $\mp 0.008$ \\
$\eta_{\mathrm{b}}$ & $\mp 0.014$ \\
$\delta^{+}$ & $\mp 0.013$ \\
$\eta_{\mathrm{c}}$ & $\mp 0.004$ \\
$\tau_{\mathrm{B}_{\mathrm{d}}}$ & \pm 0.005 \\
$\tau_{\mathrm{B}}-\tau_{\mathrm{B}_{\mathrm{d}}}$ & $\mp 0.002$ \\
$\tau_{\mathrm{B}_{\mathrm{s}}}$ & \pm 0.006 \\
$f^{\text {comb }}$ & \pm 0.002 \\
charge correlation & $\mp 0.004$ \\
Proper decay time resolution & \pm 0.003 \\
\hline & \pm 0.003 \\
\hline Total & +0.029 \\
\hline
\end{tabular}

Table 3: Systematic uncertainties of $\Delta m_{\mathrm{d}}$ for the $\mathrm{D}^{*} / \ell$ analysis. Each $\Delta m_{\mathrm{d}}$ uncertainty is signed by the direction of the correlation with the parameter; a positive correlation is denoted by \pm and a negative correlation by $\mp$. 
Adding in quadrature all systematic uncertainties, we find:

$$
\Delta m_{\mathrm{d}}=0.567 \pm 0.089(\text { stat })_{-0.023}^{+0.029}(\text { syst }) \mathrm{ps}^{-1}
$$

\section{The $\mathrm{D}^{*+} \ell^{-} / \mathrm{Q}_{\mathrm{J}}$ Analysis}

We also measure the $\mathrm{B}_{\mathrm{d}}^{0}$ oscillation frequency using reconstructed $\mathrm{D}^{*+}$ mesons and leptons in the same hemisphere, and a jet charge technique to determine the production flavor of the $\mathrm{B}_{\mathrm{d}}^{0}$.

The requirement of a lepton of the correct charge correlation in the same jet with the $\mathrm{D}^{*+}$ eliminates almost completely the background from $c \bar{c}$ events and reduces significantly $\mathrm{B}_{\mathrm{s}}^{0}$ and combinatorial background. The reduction in combinatorial background allows the use of an additional $\mathrm{D}^{0}$ decay channel, thus increasing statistics.

The analysis is almost identical to that which is described in reference [2] and has been updated to include data taken during 1994 . We briefly describe the technique below and refer the reader to reference [2] for a more detailed description.

\subsection{Reconstruction of the $B_{d}^{0}$ Meson}

The $\mathrm{B}_{\mathrm{d}}^{0}$ is detected in the channel $\mathrm{B}_{\mathrm{d}}^{0} \rightarrow \mathrm{D}^{*-} \ell^{+} \nu(\mathrm{X})$, where the $\mathrm{D}^{*-}$ is reconstructed in the decay mode $\mathrm{D}^{*-} \rightarrow \overline{\mathrm{D}}^{0} \pi^{-}$. The $\mathrm{D}^{0}$ candidates are identified in two separate decay modes: $\mathrm{D}^{0} \rightarrow \mathrm{K}^{-} \pi^{+}$and $\mathrm{D}^{0} \rightarrow \mathrm{K}^{-} \pi^{+} \pi^{0}$, where the $\pi^{0}$ is not reconstructed. For the latter process, called the satellite channel, the $\mathrm{D}^{0}$ decay is not fully reconstructed and gives rise to a broad peak in the $\mathrm{K}^{-} \pi^{+}$invariant mass distribution about $250 \mathrm{MeV}$ below the $\mathrm{D}^{0}$ mass. For the fully reconstructed channel we select candidates in the invariant mass range $1.79 \mathrm{GeV}<M\left(\mathrm{~K}^{-} \pi^{+}\right)<1.94 \mathrm{GeV}$ and for the satellite channel we select candidates in the range $1.41 \mathrm{GeV}<M\left(\mathrm{~K}^{-} \pi^{+}\right)<1.77 \mathrm{GeV}$.

The $\mathrm{D}^{0}$ vertex is formed from a fit using the three tracks forming the $\mathrm{D}^{*+}$ candidate, as is done for the $D^{*} / \ell$ analysis.

The $\mathrm{D}^{*+}$ candidates are combined with a lepton track in the same jet. Electron candidates are required to have momentum greater than $2 \mathrm{GeV}$ and muon candidates are required to have momentum greater than $3 \mathrm{GeV}$. In addition, they are required to have $p_{t}>0.6 \mathrm{GeV}$. In order to suppress combinatorial background, the invariant mass of the $\mathrm{D}^{*+} \ell^{-}$system is required to be in the range $2.8-5.3 \mathrm{GeV}$.

The charge of the lepton gives the $b$ flavor at the decay time and a jet charge 
technique is used to tag the production flavor. The jet charge is defined as

$$
Q_{j e t}=\sum_{i=1}^{n} q_{i} \cdot\left(\frac{p_{l, i}}{E_{\text {beam }}}\right)^{\kappa},
$$

where the sum runs over all charged tracks in the jet. The jet charges of two jets are used: the jet containing the $\mathrm{B}_{\mathrm{d}}^{0}$ candidate and the most energetic other jet (opposite jet). The quantity

$$
Q_{2 j e t}=Q_{j e t}^{\kappa=0}\left(B_{\mathrm{d}}^{0}\right)-10 \cdot Q_{j e t}^{\kappa=1}(o p p),
$$

where $Q_{j e t}\left(\mathrm{~B}_{\mathrm{d}}^{0}\right)$ and $Q_{j e t}(o p p)$ are the jet charges of the $\mathrm{B}_{\mathrm{d}}^{0}$ jet and the opposite jet, respectively, provides good discrimination between $\mathrm{B}_{\mathrm{d}}^{0}$ and $\overline{\mathrm{B}}_{\mathrm{d}}^{0}$ at production. Events for which $Q_{2 j e t}$ and the lepton have the same charge (like-sign) are tagged as unmixed and those for which $Q_{2 j e t}$ and the lepton have opposite charge (unlike-sign) are tagged as mixed. To reject events with poor flavor discrimination it is required that $\left|Q_{2 j e t}\right|>1$. From Monte Carlo studies we predict a mistag rate of 0.28 using this method. This value found from Monte Carlo simulated events is not used in the analysis. Instead, the mistag rate is obtained directly from the data as a free parameter in the fit for $\Delta m_{\mathrm{d}}$. The fitted value is compared to the Monte Carlo prediction as a consistency check.

\begin{tabular}{|l|c|c|}
\hline Decay mode & Number of $\mathrm{D}^{*+} \ell^{-}$candidates & Background \\
\hline $\mathrm{D}^{0} \rightarrow \mathrm{K}^{-} \pi^{+}$ & 406 & $49 \pm 7$ \\
$\mathrm{D}^{0} \rightarrow \mathrm{K}^{-} \pi^{+} \pi^{0}$ & 794 & $225 \pm 15$ \\
\hline Sum & 1200 & $274 \pm 17$ \\
\hline
\end{tabular}

Table 4: Number of selected $\mathrm{D}^{*+} \ell^{-}$candidates and estimated background.

Figure 9 shows the distributions of the mass difference $\delta_{M}=M\left(\mathrm{D}^{*}\right)-M\left(\mathrm{D}^{0}\right)$ for candidates in the fully reconstructed and satellite channels. The shape of the $\delta_{M}$ distribution for the combinatorial background is determined from the data using a reflected soft pion technique, described in detail in [16]. A background sample free of the correlations between the slow pion and $\mathrm{D}^{0}$ tracks which form a peak in the $\delta_{M}$ spectrum is obtained by combining:

- wrong charge candidates, reconstructed by combining a $\mathrm{D}^{* \pm}$ candidate with a lepton of the same charge as the $\mathrm{D}^{* \pm}$;

- reflected pion candidates, constructed by selecting a slow pion candidate track from the hemisphere opposite a normal $\mathrm{D}^{0}$ candidate and combining them to form a $\mathrm{D}^{* \pm}$ candidate after reflecting the pion through the origin. 
The shape of the background is parametrized in a fit to the $\delta_{M}$ distribution using the following empirical functional form:

$$
A \mathrm{e}^{-B \delta_{M}}\left(\frac{\delta_{M}}{m_{\pi}}-1\right)^{C},
$$

where $A, B$ and $C$ are free parameters in the fit and $m_{\pi}$ is the pion mass. To determine the number of signal and background events, the resulting background form given by the fit is normalized to the signal sideband in the $\delta_{M}$ mass spectrum. The sideband region is defined as $0.17<\delta_{M}<0.25 \mathrm{GeV}$. The signal region is defined as $\delta_{M}<0.15$ $\mathrm{GeV}$ for the fully reconstructed channel and $\delta_{M}<0.16 \mathrm{GeV}$ for the satellite channel. The number of selected $\mathrm{D}^{*+} \ell^{-}$candidates and estimated background, including the systematic error, are given in Table 4.

\subsection{Reconstruction of the $\mathrm{B}_{\mathrm{d}}^{0}$ Proper Decay Time}

The $\mathrm{B}_{\mathrm{d}}^{0}$ decay vertex is formed by extrapolating the reconstructed $\mathrm{D}^{*+}$ momentum vector from the $\mathrm{D}^{0}$ vertex to the intersection with the lepton track. The $\mathrm{B}_{\mathrm{d}}^{0}$ decay length, $L$, is calculated from a fit to the estimated primary vertex and the $\mathrm{B}_{\mathrm{d}}^{0}$ decay vertex using the direction of the visible $\mathrm{D}^{*+} \ell^{-}$momentum as a constraint.

The boost, $\beta \gamma$, is parameterized as a function of the momentum and the invariant mass of the $\mathrm{D}^{*+} \ell^{-}$pair, $p_{\mathrm{D}^{*} \ell}$ and $m_{D^{*} \ell}$, respectively. The parameterization takes the form

$$
\beta \gamma=\frac{p_{\mathrm{D}^{*} \ell}}{m_{\mathrm{B}}} \cdot s\left(p_{\mathrm{D}^{*} \ell}, m_{D^{*} \ell}\right),
$$

where $m_{\mathrm{B}}=5.279 \mathrm{GeV}$ is the mass of the $\mathrm{B}_{\mathrm{d}}^{0}$ meson and $s$ is a factor that corrects for the missing energy carried by the undetected $\nu$, and also the $\pi^{0}$ in the case of the satellite channel. Monte Carlo simulated $\mathrm{B}_{\mathrm{d}}^{0}$ decays are used to estimate $s$ separately for the fully reconstructed and satellite channels in eleven bins of $p_{\mathrm{D}^{*} \ell}$ and $m_{D^{*} \ell}$.

The decay length and boost estimates are combined to form the proper decay time, $t$, of the $\mathrm{B}_{\mathrm{d}}^{0}$. The average fractional resolution on $t$ ranges from $16 \%$ to $20 \%$, depending on the kinematics of the $D^{*+} \ell^{-}$pair.

\subsection{Measurement of $\Delta m_{\mathrm{d}}$}

The $D^{*+} \ell^{-} / Q_{\mathrm{J}}$ events are binned in proper decay time and the $\mathrm{B}_{\mathrm{d}}^{0}$ oscillation frequency is measured from the time distribution of the ratio

$$
R(t)=\frac{N_{\text {like }}(t)-N_{\text {like }}^{\mathrm{bck}}(t)}{N_{\text {tot }}(t)-N_{\text {tot }}^{\mathrm{bck}}(t)}
$$


where $N_{\text {tot }}(t)$ and $N_{\text {tot }}^{\text {bck }}(t)$ are the total number of candidates and estimated combinatorial background, and $N_{\text {like }}(t)$ and $N_{\text {like }}^{\text {bck }}(t)$ are the corresponding numbers for the the like-sign events. The proper decay time distribution of the combinatorial background is estimated using events in the $\delta_{M}$ sideband region $0.17-0.25 \mathrm{GeV}$. As described in section 3.3, we assume that $(16 \pm 9) \%[13]$ of the $\mathrm{D}^{*+}$ signal is from $\mathrm{B}^{-}$decays. For this sample, the fraction of events from $\mathrm{B}_{\mathrm{s}}^{0}$ decays is negligible and is ignored. The expected distribution of $R(t)$ is

$$
R(t)=\eta_{\mathrm{b}}+\frac{1-2 \eta_{\mathrm{b}}}{1+N_{+}(t) / N_{0}(t)} \cdot \sin ^{2}\left(\Delta m_{\mathrm{d}} \cdot t / 2\right),
$$

where $N_{+}(t)$ and $N_{0}(t)$ are the number of $\mathrm{B}^{-}$and $\mathrm{B}_{\mathrm{d}}^{0}$ which decay at time $t$ and $\eta_{\mathrm{b}}$ is the mistag rate. The ratio $N_{+}(t) / N_{0}(t)$ can be expressed as

$$
\frac{N_{+}(t)}{N_{0}(t)}=\frac{N_{+}(0)}{N_{0}(0)} \cdot e^{\left(t / \tau_{\mathrm{B}_{\mathrm{d}}}\right) \cdot\left(\delta \tau / \tau_{\mathrm{B}}-\right)}
$$

where $\delta \tau=\tau_{\mathrm{B}^{-}}-\tau_{\mathrm{B}_{\mathrm{d}}}$ and $N_{+}(0)$ and $N_{0}(0)$ are the total number of $\mathrm{B}^{-}$and $\mathrm{B}_{\mathrm{d}}^{0}$ decays, respectively, in the sample.

The distribution of $R(t)$ is shown in Figure 10 and is fitted to the functional form of equations (4) and (5) by minimizing the $\chi^{2}$, with $\Delta m_{\mathrm{d}}$ and $\eta_{\mathrm{b}}$ as free parameters. The last bin in Figure 10 is chosen to be twice as large as the other bins in order to offset the low statistics at large proper decay time. The point is centered at the mean of the expected distribution of events in that bin. For all other bins, the points are placed at the bin centers, but differ negligibly from the expected mean positions. The fit gives a $\chi^{2}$ of 4.4 with 7 degrees of freedom and returns:

$$
\Delta m_{\mathrm{d}}=0.539 \pm 0.060 \mathrm{ps}^{-1}
$$

and

$$
\eta_{\mathrm{b}}=0.277 \pm 0.023
$$

The fitted value of $\eta_{\mathrm{b}}$ is consistent with the prediction of 0.28 from Monte Carlo simulated events.

\subsection{Study of Systematic Uncertainties}

The following systematic uncertainties have been estimated:

- The $\mathbf{B}^{-}$fraction: The error due to the uncertainty in the fraction of $\mathrm{B}^{-}$in the $D^{*+} \ell^{-} / Q_{\mathrm{J}}$ sample is evaluated by changing this fraction within the range $(16 \pm 9) \%$ and repeating the fit. The variation in $\Delta m_{\mathrm{d}}$ is $\pm 0.019 \mathrm{ps}^{-1}$.

- The B meson lifetimes: The $\mathrm{B}_{\mathrm{d}}^{0}$ lifetime is varied within its uncertainty while keeping the lifetime difference between $\mathrm{B}^{-}$and $\mathrm{B}_{\mathrm{d}}^{0}$ constant. This changes $\Delta m_{\mathrm{d}}$ by less than $0.001 \mathrm{ps}^{-1}$. Varying the lifetime difference, $\delta \tau$, within its uncertainty results in a change in $\Delta m_{\mathrm{d}}$ of $\pm 0.007 \mathrm{ps}^{-1}$. 
- The combinatorial background fraction: The error on the estimated combinatorial background was computed taking into account the statistical error on the number of background events, the error due to the normalization of the sideband to that in the background estimator sample, and the error due to the background shape uncertainty, which was estimated using an alternative parameterization. The resulting estimated combinatorial background error of $6 \%$ leads to a \pm 0.003 $\mathrm{ps}^{-1}$ systematic error on $\Delta m_{\mathrm{d}}$.

- The proper decay time resolution: The systematic error due to proper decay time resolution is estimated by performing the fit on Monte Carlo simulated events which include smearing of the proper decay time and comparing the fit result with the value of $\Delta m_{\mathrm{d}}$ used to generate the events. This procedure also covers any systematic effect due to the choice of bin centers used in the fit. The systematic uncertainty due to proper decay time resolution is estimated to be $\pm 0.012 \mathrm{ps}^{-1}$.

\begin{tabular}{|l|c|}
\hline Source of error & Error on $\Delta m_{\mathrm{d}}\left(\mathrm{ps}^{-1}\right)$ \\
\hline $\mathrm{B}^{-}$fraction & \pm 0.019 \\
$\delta \tau$ & \pm 0.007 \\
Comb. background fraction & \pm 0.003 \\
Proper decay time resolution & \pm 0.012 \\
\hline Total & \pm 0.024 \\
\hline
\end{tabular}

Table 5: Systematic uncertainties of $\Delta m_{\mathrm{d}}$ for the $\mathrm{D}^{*+} \ell^{-} / \mathrm{Q}_{\mathrm{J}}$ analysis. Each $\Delta m_{\mathrm{d}}$ uncertainty is signed by the direction of the correlation with the parameter; a positive correlation is denoted by \pm and a negative correlation by $\mp$.

The systematic uncertainties are summarized in Table 5. Adding in quadrature all of the systematic uncertainties listed above, the final result is

$$
\Delta m_{\mathrm{d}}=0.539 \pm 0.060(\text { stat }) \pm 0.024(\text { syst }) \mathrm{ps}^{-1} .
$$

\section{Combined Result and Conclusions}

We have reported on two measurements of the $\mathrm{B}_{\mathrm{d}}^{0}$ oscillation frequency, $\Delta m_{\mathrm{d}}$, using events with reconstructed $\mathrm{D}^{*+}$ mesons and leptons. The data were collected with the OPAL detector during 1990-1994. The first measurement uses a sample of $348 \mathrm{D}^{*+}$ candidates with a lepton in the opposite hemisphere, of which $167 \pm 25$ are expected to come from $\mathrm{B}_{\mathrm{d}}^{0}$ decays. We find

$$
\Delta m_{\mathrm{d}}=0.567 \pm 0.089(\text { stat })_{-0.023}^{+0.029}(\text { syst }) \mathrm{ps}^{-1} .
$$


This value is consistent with and supersedes the previous OPAL measurement using $\mathrm{D}^{*+}$ mesons and leptons in opposite hemispheres [1].

We have also updated a measurement of $\Delta m_{\mathrm{d}}$ using $\mathrm{D}^{*+}$ and lepton candidates in the same hemisphere and a jet charge technique. From a sample of $1200 \mathrm{D}^{*+} \ell^{-}$ candidates, of which $778 \pm 84$ are expected to be from $\mathrm{B}_{\mathrm{d}}^{0}$ decays, we find

$$
\Delta m_{\mathrm{d}}=0.539 \pm 0.060(\text { stat }) \pm 0.024(\text { syst }) \mathrm{ps}^{-1} .
$$

This value supersedes that of [2].

There is virtually no statistical correlation between the two measurements. There are only 5 events in common between the two samples, and even for these events each method estimates the $B_{d}^{0}$ proper decay time and production flavor by using different quantities extracted from the events. The combined result is

$$
\Delta m_{\mathrm{d}}=0.548 \pm 0.050(\text { stat })_{-0.019}^{+0.023}(\text { syst }) \mathrm{ps}^{-1}
$$

which is compatible with other measurements of $\Delta m_{\mathbf{d}}[12,17]$. The systematic effects that are assumed to be correlated are the $B_{d}^{0}$ lifetime, the difference between the $B_{d}^{0}$ and $\mathrm{B}^{-}$lifetimes, and the $\mathrm{B}^{-}$fraction in the sample.

\section{Acknowledgements}

It is a pleasure to thank the SL Division for the efficient operation of the LEP accelerator, the precise information on the absolute energy, and their continuing close cooperation with the experimental group. In addition to the support staff at our own institutions we are pleased to acknowledge the

Department of Energy, USA,

National Science Foundation, USA,

Particle Physics and Astronomy Research Council, UK,

Natural Sciences and Engineering Research Council, Canada,

Israel Ministry of Science,

Israel Science Foundation, administered by the Israel Academy of Science and Humanities,

Minerva Gesellschaft,

Japanese Ministry of Education, Science and Culture (the Monbusho) and a grant under the Monbusho International Science Research Program,

German Israeli Bi-national Science Foundation (GIF),

Direction des Sciences de la Matière du Commissariat à l'Energie Atomique, France, Bundesministerium für Bildung, Wissenschaft, Forschung und Technologie, Germany, National Research Council of Canada, Hungarian Foundation for Scientific Research, OTKA T-016660, and OTKA F-015089. 


\section{References}

[1] OPAL Collaboration, R. Akers et al., Phys. Lett. B 336 (1994) 585.

[2] OPAL Collaboration, R. Akers et al., Phys. Lett. B 327 (1994) 411.

[3] OPAL Collaboration, K. Ahmet et al., Nucl. Instrum. Methods A 305 (1991) 275.

[4] P. P. Allport et al., Nucl. Instrum. Methods A 324 (1993) 34;

P. P. Allport et al., Nucl. Instrum. Methods A 346 (1994) 476.

[5] OPAL Collaboration, G. Alexander et al., Z. Phys. C 52 (1991) 175.

[6] JADE Collaboration, W. Bartel et al., Z. Phys. C 33 (1986) 23;

JADE Collaboration, S. Bethke et al., Phys. Lett. B 213 (1988) 235.

[7] T. Sjöstrand, Comp. Phys. Comm. 39 (1986) 347;

T. Sjöstrand and M. Bengtsson, Comp. Phys. Comm. 43 (1987) 367;

T. Sjöstrand, PYTHIA 5.7 and JETSET 7.4: Physics and Manual, CERN-TH $7112 / 93$

T. Sjöstrand, Comp. Phys. Com. 82, (1994) 74.

[8] The JETSET 7.4 parameters were tuned as described in

OPAL Collaboration, G. Alexander et al., Z. Phys. C 69 (1996) 543.

[9] J.Allison et al., Nucl. Instrum. Methods A 317 (1992) 47.

[10] OPAL Collaboration, CERN-PPE/95-179, to be published in Z. Phys. C.

[11] OPAL Collaboration, R. Akers et al., Z. Phys. C 65 (1995) 17.

[12] OPAL Collaboration, R. Akers et al., Z. Phys. C 66 (1995) 555.

[13] OPAL Collaboration, P. D. Acton et al., Phys. Lett. B 307 (1993) 247.

[14] The LEP B Lifetime Working Group has used measurements from the following papers to calculate the average $\mathrm{B}_{\mathrm{d}}^{0}$ and $\mathrm{B}^{-}$lifetimes, which will appear in the 1996 Particle Data Group Review of Particle Properties:

ALEPH Collaboration, D. Buskulic et al., Z. Phys. C 69 (1966) 585;

CDF Collaboration, F. Abe et al., Phys. Rev. Lett. C 72 (1994) 3456;

CDF Collaboration, F. Abe et al., submitted to Phys. Rev. Lett.; DELPHI Collaboration, P. Abreu et al., Z. Phys. C 68 (1995) 13;

DELPHI Collaboration, W. Adam et al., Z. Phys. C 68 (1995) 363;

OPAL Collaboration, R. Akers et al., Z. Phys. C 67 (1995) 379.

[15] The LEP B Lifetime Working Group has used measurements from the following papers to calculate the average $\mathrm{B}_{\mathrm{s}}^{0}$ lifetime, which will appear in the 1996 Particle Data Group Review of Particle Properties:

ALEPH Collaboration, D. Buskulic et al., Phys. Lett. B 361 (1995) 221; 
ALEPH Collaboration, D. Buskulic et al., Z. Phys. C 69 (1996) 585;

CDF Collaboration, F. Abe et al., Phys. Rev. Lett. C 74 (1995) 4988;

DELPHI Collaboration, P. Abreu et al., CERN-PPE/96-32, submitted to Z. Phys.

$\mathrm{C}$;

OPAL Collaboration, R. Akers et al., Phys. Lett. B 350 (1995) 273.

[16] OPAL Collaboration, R. Akers et al., Z. Phys. C 67 (1995) 27.

[17] ALEPH Collaboration, D. Buskulic et al., Phys. Lett. B 313 (1993) 498;

ALEPH Collaboration, D. Buskulic et al., Phys. Lett. B 322 (1994) 441;

DELPHI Collaboration, P. Abreu et al., Phys. Lett. B 338 (1994) 409;

DELPHI Collaboration, P. Abreu et al., CERN-PPE/96-06, submitted to Z. Phys. C. 


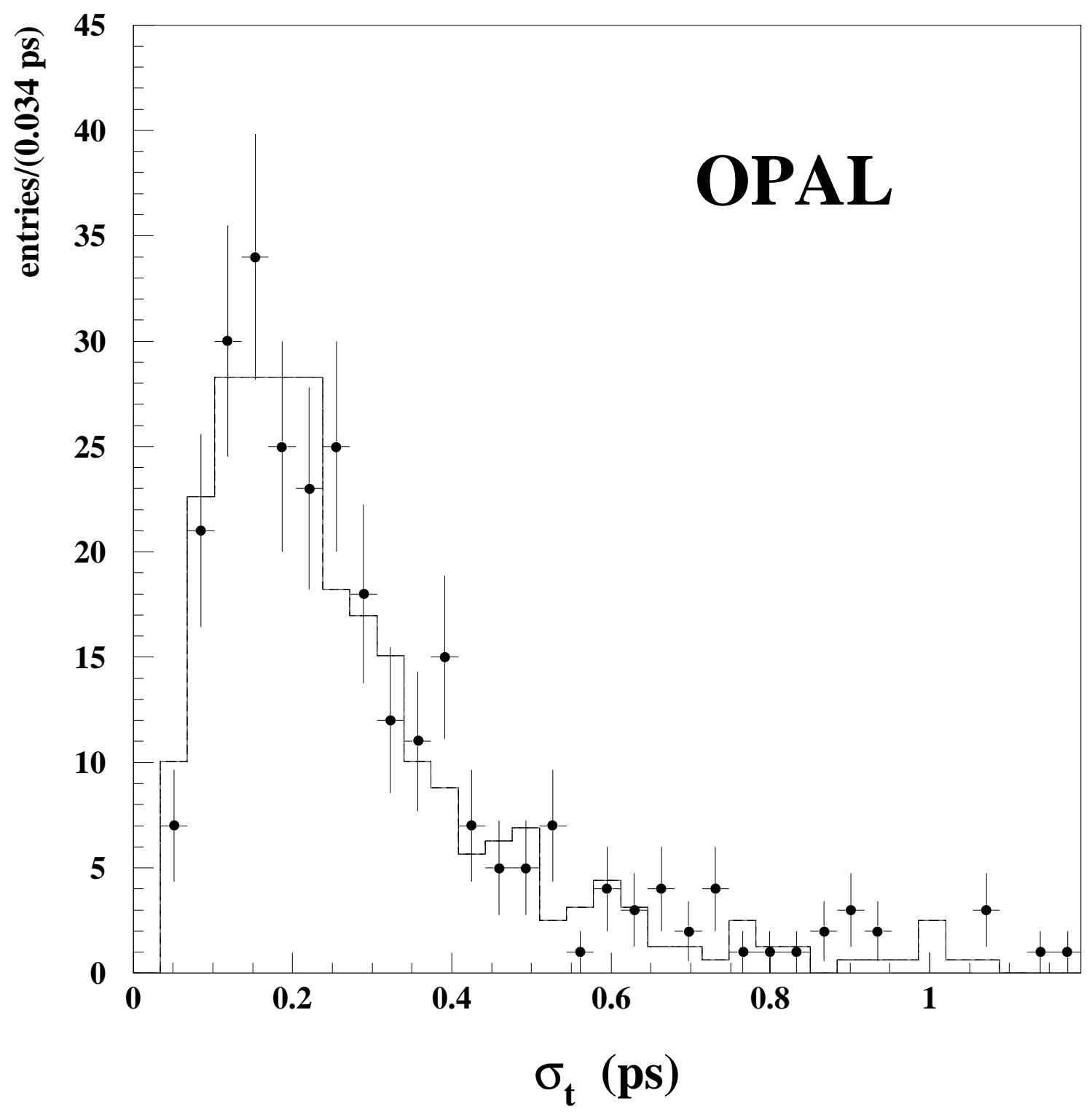

Figure 1: Distribution of measured $\sigma_{t}$ for data (points) and Monte Carlo simulated events (histogram) for the $D^{*} / \ell$ analysis. 


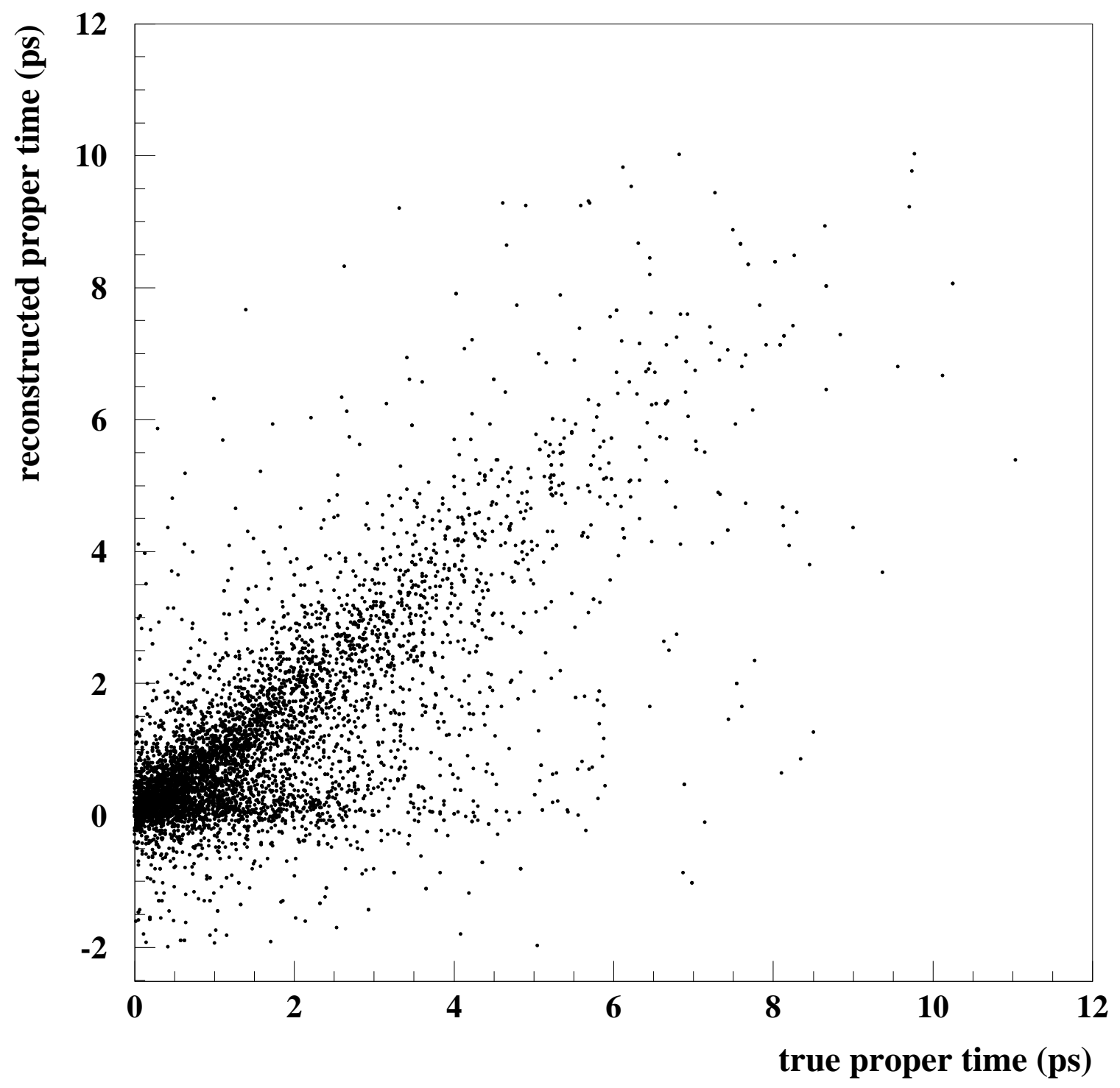

Figure 2: The reconstructed proper decay time versus the true proper decay time for $\mathrm{D}^{*} / \ell$ events in Monte Carlo simulated $\mathrm{B}_{\mathrm{d}}^{0}$ decays. 

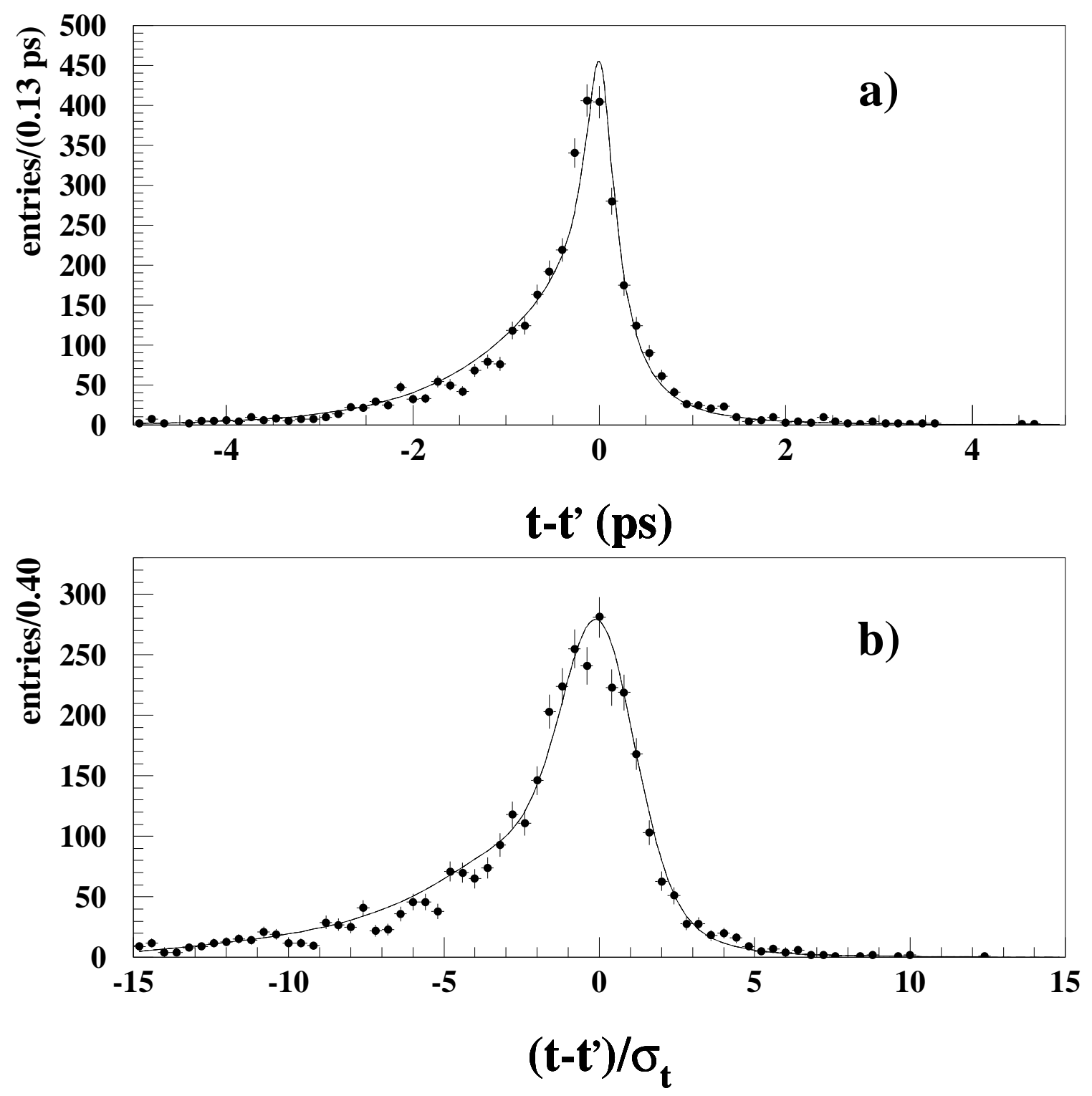

Figure 3: a) The difference between the reconstructed proper decay time $t$ and the true proper decay time $t^{\prime}$, and $\mathrm{b}$ ) the same quantity divided by the calculated error on $t$, for $\mathrm{D}^{*} / \ell$ events in Monte Carlo simulated $\mathrm{B}_{\mathrm{d}}^{0}$ decays. 


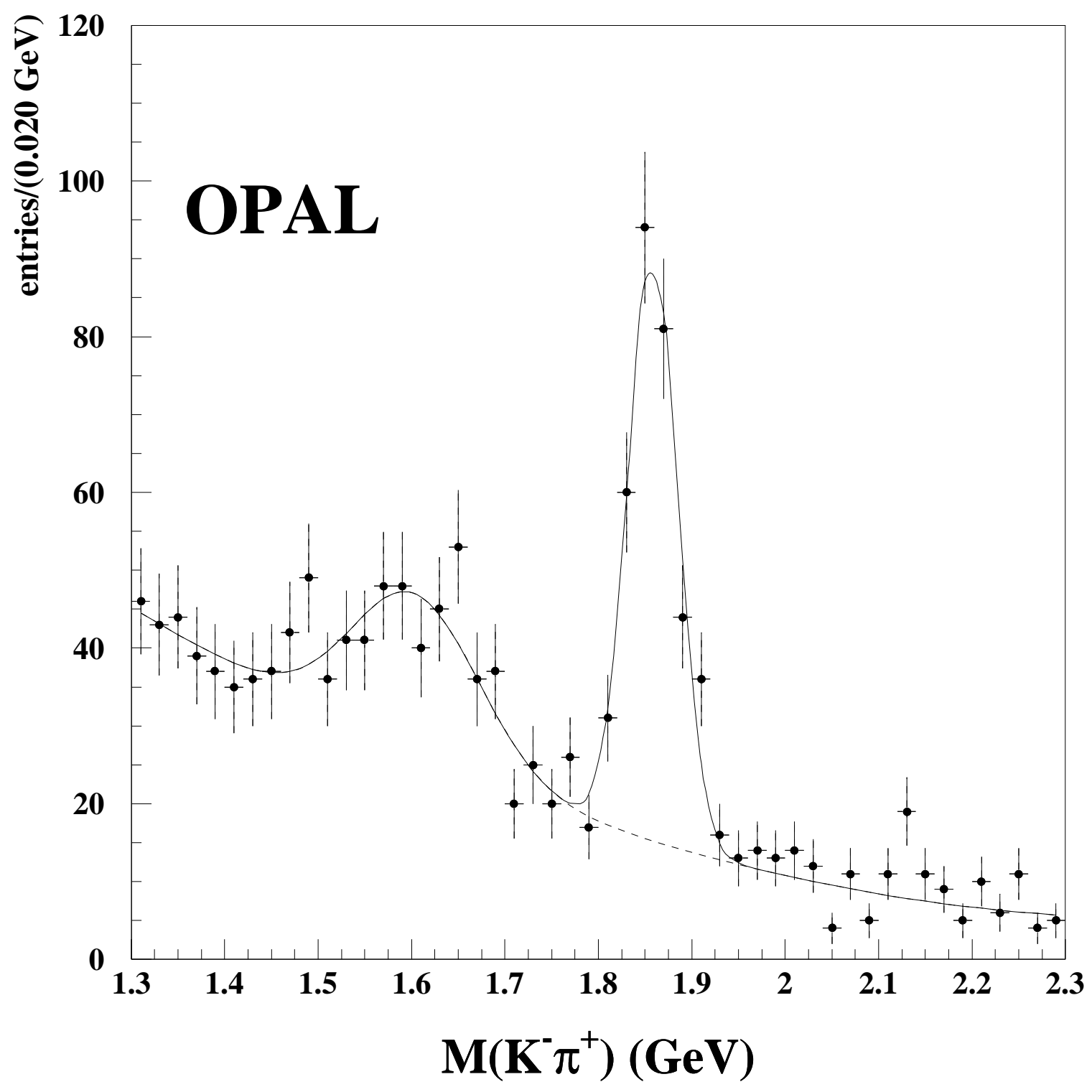

Figure 4: The reconstructed $\mathrm{K}^{-} \pi^{+}$invariant mass distribution for selected $\mathrm{D}^{*} / \ell$ candidates. The solid curve is the fit to signal and background events and the dashed curve is the background fit alone. 

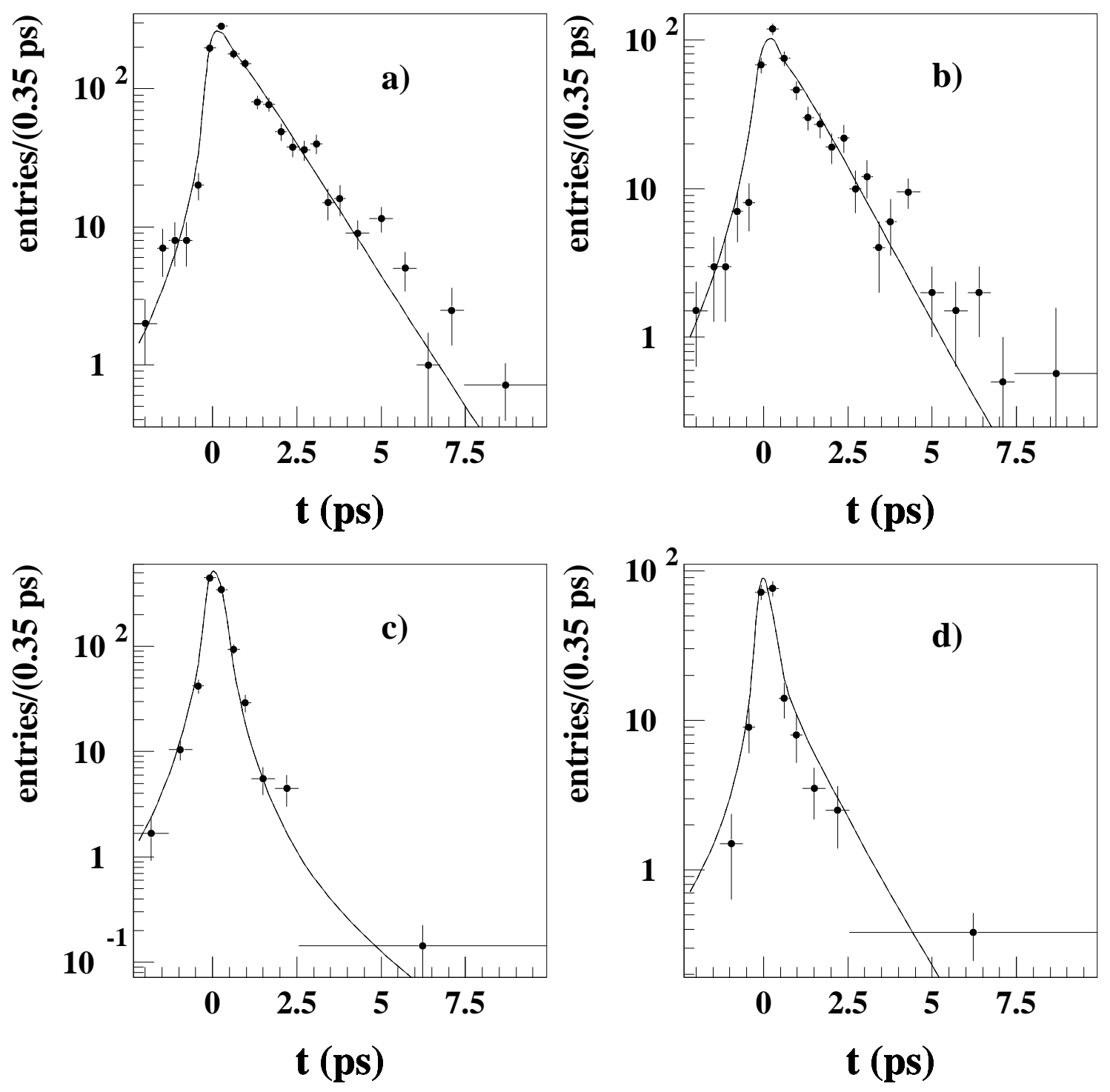

Figure 5: The reconstructed proper decay time for a) Monte Carlo simulated $\mathrm{B}_{\mathrm{d}}^{0}$ decays, b) Monte Carlo simulated $\mathrm{B}^{-}$decays, c) Monte Carlo simulated $\mathrm{Z} \rightarrow \mathrm{c} \overline{\mathrm{c}}$ events and d) combinatorial background from the sideband of the $\mathrm{D}^{0}$ mass distribution in the data. The curves represent the parametrized shapes. The distributions are for the $\mathrm{D}^{*} / \ell$ analysis. 


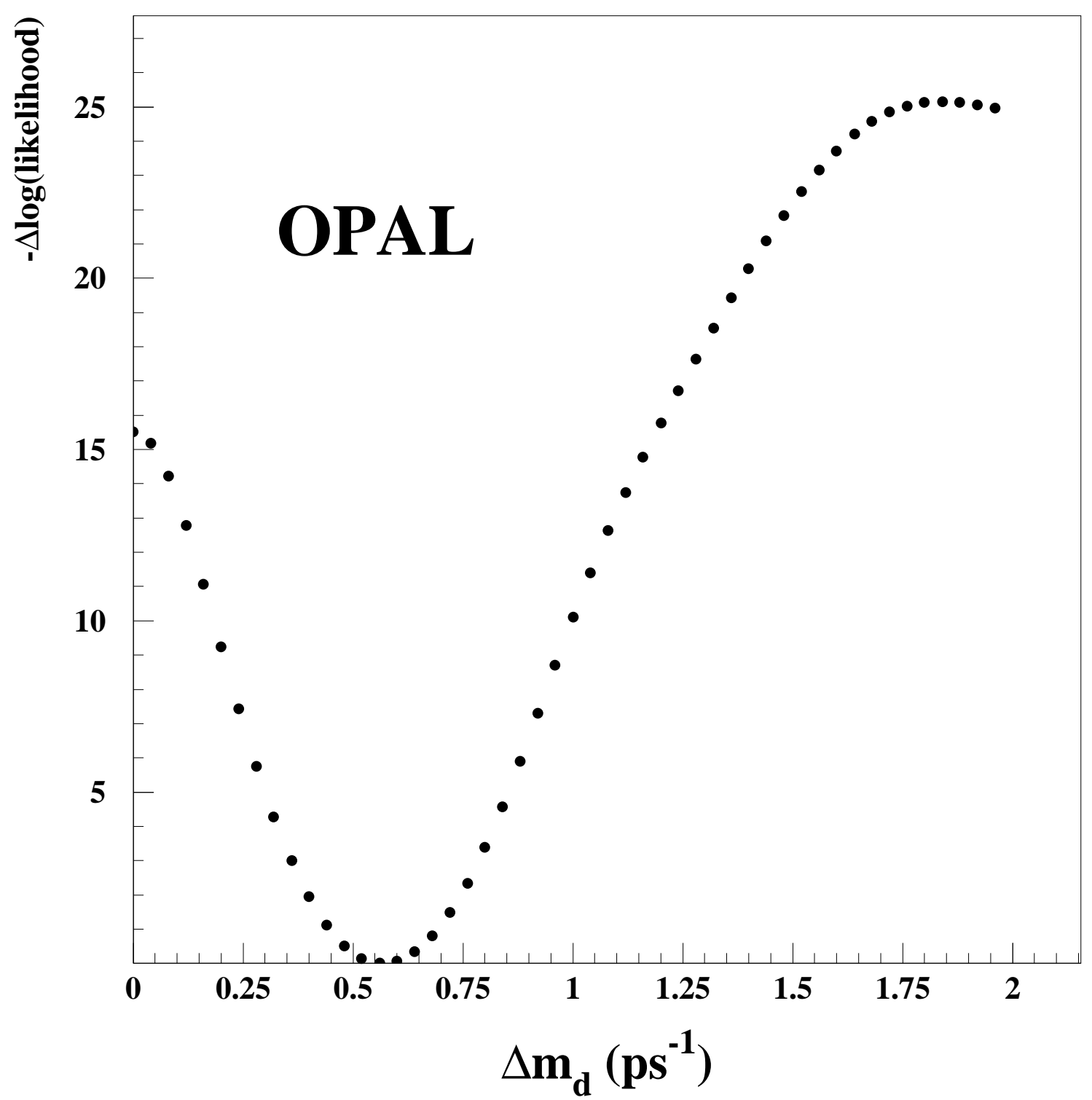

Figure 6: The difference between $-\log \mathcal{L}$ and its minimum value as a function of $\Delta m_{\mathrm{d}}$ for the $\mathrm{D}^{*} / \ell$ analysis. 

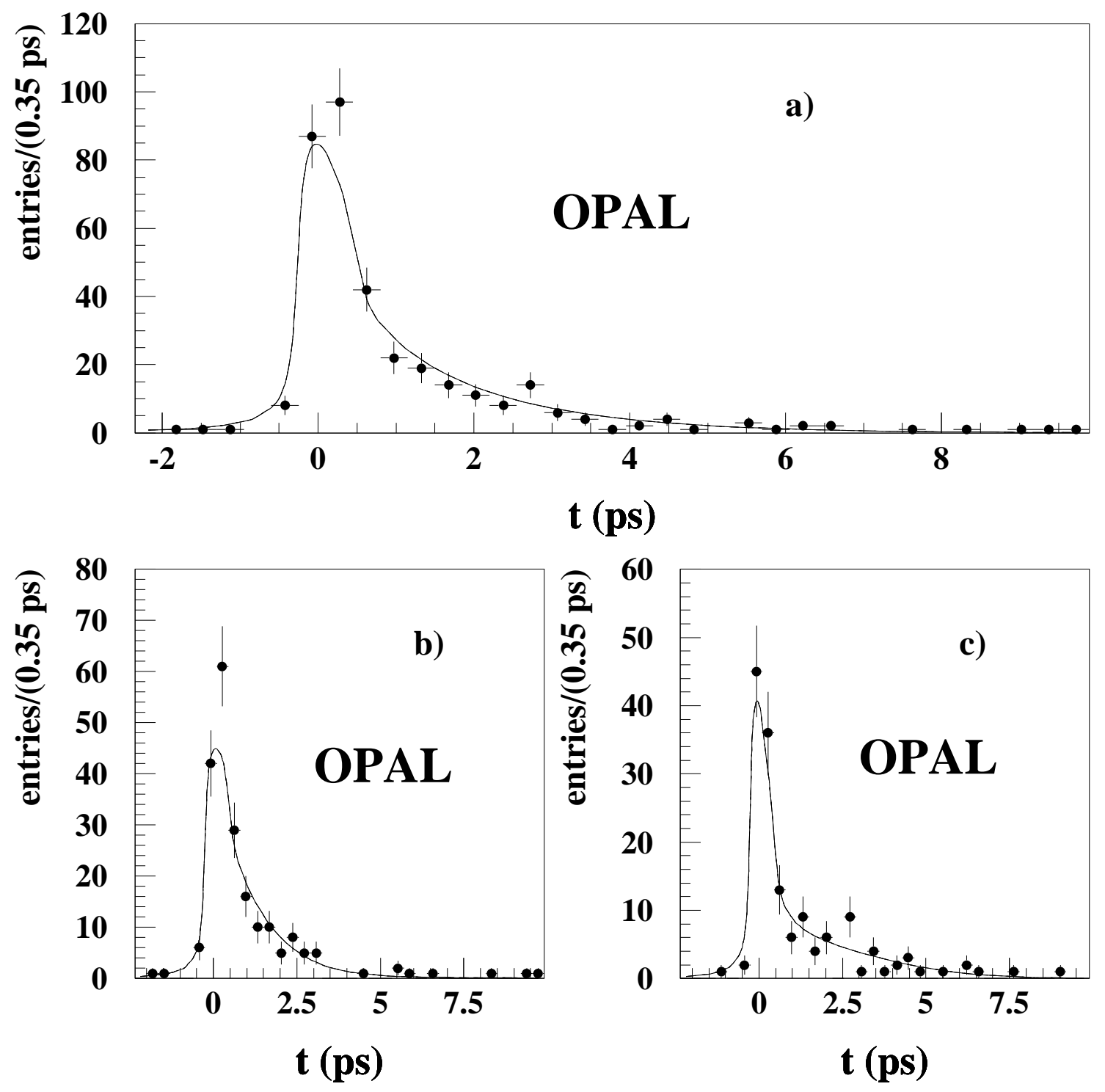

Figure 7: The reconstructed proper decay time distributions for selected $D^{*} / \ell$ events: a) all events, b) like-sign events $\left(D^{*+} / \ell^{+}\right)$and c) unlike-sign events $\left(D^{*+} / \ell^{-}\right)$. The curves represent the shapes predicted by the fit. 


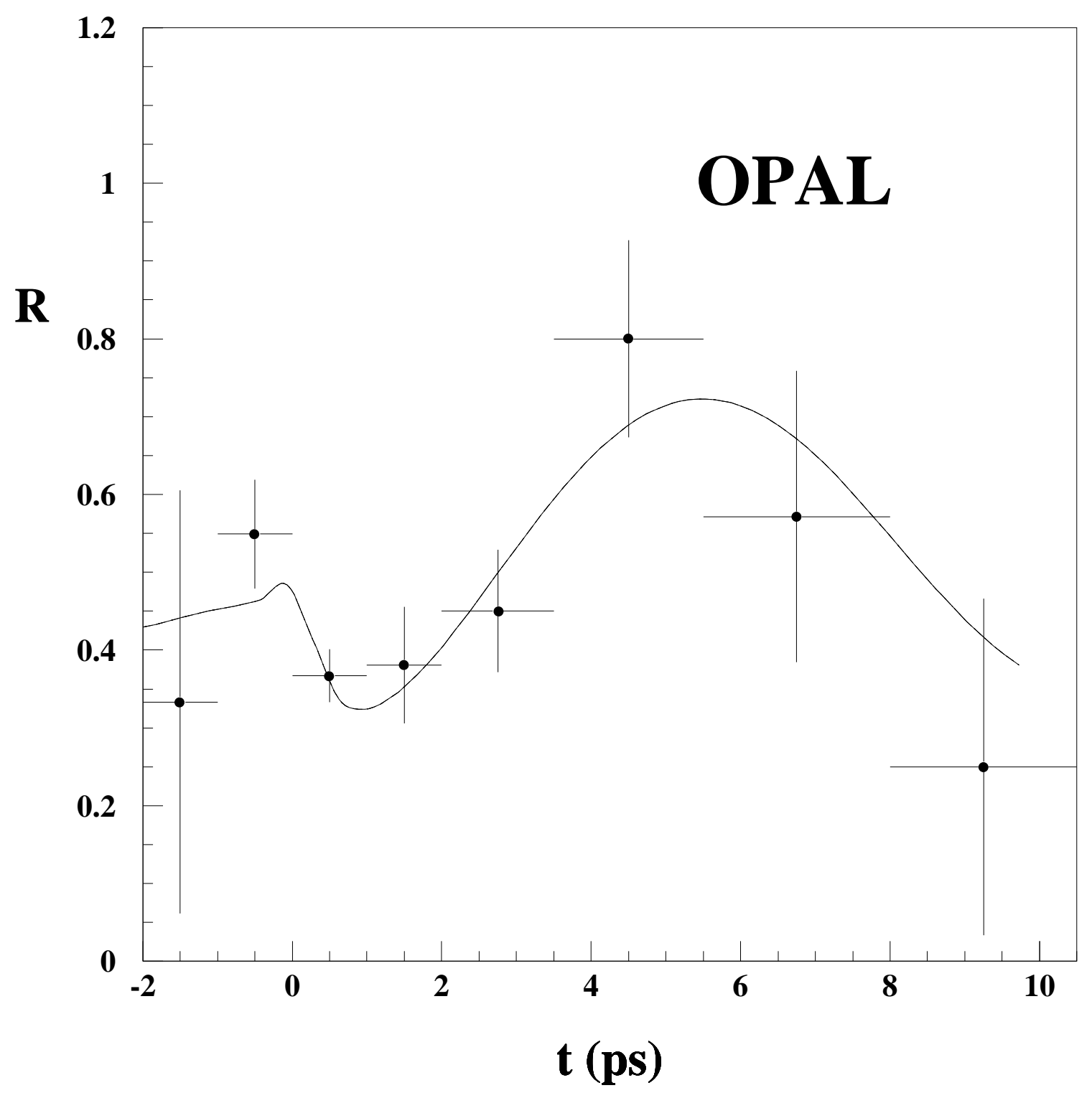

Figure 8: The ratio, R, of like-sign events to total events, as a function of reconstructed proper decay time, for $D^{*} / \ell$ events. The curve shows the result of the fit. 

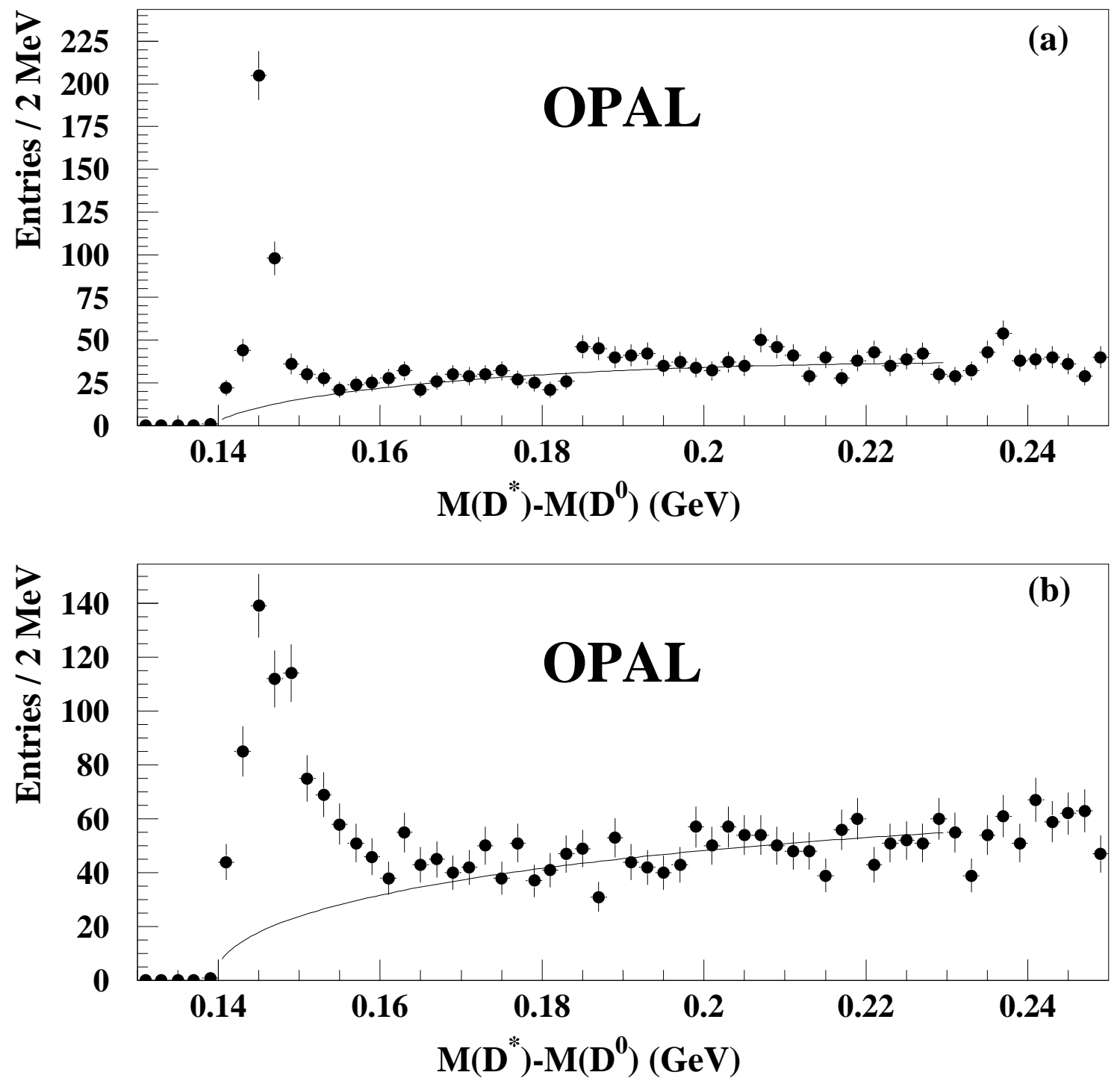

Figure 9: Distributions of $M\left(\mathrm{D}^{*+}\right)-M\left(\mathrm{D}^{0}\right)$ for a) $\mathrm{D}^{0} \rightarrow \mathrm{K}^{-} \pi^{+}$events and b) satellite events, for selected $D^{*+} \ell^{-} / Q_{\mathrm{J}}$ events. The curves represent the estimated background. 


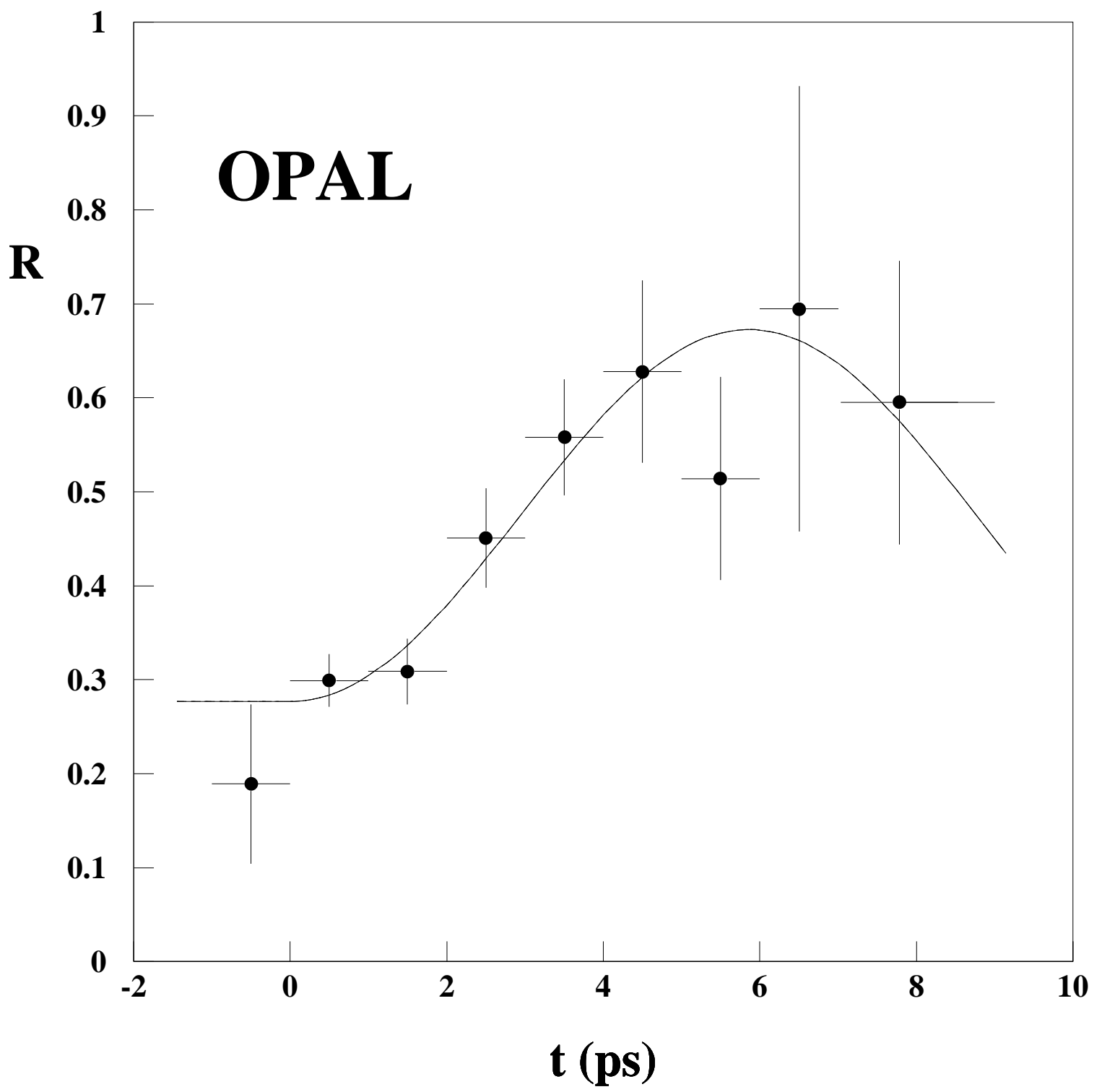

Figure 10: The ratio, $\mathrm{R}$, of like-sign events to total events, as a function of reconstructed proper decay time, for selected $\mathrm{D}^{*+} \ell^{-} / \mathrm{Q}_{\mathrm{J}}$ events. The curve shows the result of the fit. 